\title{
A Simple Passive Device for the Drag Reduction of an Ahmed Body
}

\author{
N. A. Siddiqui ${ }^{\dagger}$ and M. A. Chaab \\ Ontario Tech University, Oshawa, Ontario, L1G0C5, Canada \\ †Corresponding Author Email: naseeb.siddiqui@ontariotechu.net
}

(Received May 9, 2020; accepted July 9, 2020)

\begin{abstract}
In this paper, a simple passive device is proposed for drag reduction on the $35^{\circ}$ Ahmed body. The device is a simple rectangular flap installed at the slant surface of the model to investigate the effect of slant volume, formed between the device and the slant surface, on the flow behaviour. The slant volume can be varied by changing the flap angle. This investigation is performed using the FLUENT software at a Reynolds number of $7.8 \times 10^{5}$ based on the height of the model. The SST k-omega model is used to solve the Navier-stokes equations. It is found that this passive device influences the separation bubbles created inside the slant volume and provides a maximum drag reduction of approximately $14 \%$ at the flap angle of $10^{\circ}$. Moreover, the device delays the main separation point, which changes the flow conditions at the back of the model. The drag reduction was found to mainly dependent on the suppression of the separation bubbles formed inside the slant volume, which leads to faster pressure recovery. The cause of this pressure recovery is found to be the reduction in recirculation length and width. Also, the addition of a flap reduces the turbulent kinetic energy, which lessened the wake entrainment in the recirculation region, leading to a drag reduction. Also, it hinders the formation of horseshoe vortex that provides a pressure recovery and influence the wake width. However, the investigation also reveals that this device does not reduce the induced drag due to longitudinal vortex from the side edges.
\end{abstract}

Keywords: Ahmed body; Passive drag reduction; Bluff body; Backflow reduction; Road vehicles; Rectangular flap device; CFD.

\section{INTRODUCTION}

Greenhouse gas emissions, human health, and depletion of fossil fuel resources are necessitating efforts to minimize fuel consumption in road vehicles. (Hucho 1993) suggested that the aerodynamic drag of a mid-size car contributes up to $46 \%$ of the fuel consumption at highway speeds. (McCallen et al. 1999) stated that the drag coefficient of 0.6 of a modern tractor-trailer consumes $65 \%$ of fuel to overcome the aerodynamic drag. A $43 \%$ fuel saving is achievable if the drag coefficient of 0.6 is reduced to 0.3. Furthermore, (Hsu and Davis 2010) estimated that a $40 \%$ reduction in aerodynamic drag of trucks leads to $\$ 10,000$ savings every year per vehicle. (Kim et al. 2016) argue that the classical approach to reduce drag, such as shape modification, is not feasible in the modern world because of the aesthetic demands of the customers. Therefore, the urgency to develop new control devices for fuel reduction without compromising the aesthetics is a growing research area of interest.

In the automotive geometries, the Ahmed body proposed by (Ahmed et al. 1984) laid the foundation for understanding the flow behaviour around generic road vehicle shapes. The Ahmed body shows the critical importance of afterbody on the drag. At moderate afterbody slant angles (from $12.5^{\circ}$ to $30^{\circ}$ ), extreme pairs of counter-rotating vortices emerge in the wake of the body. Hence, it reduces the base pressure, which ultimately increases the pressure drag. Furthermore, compared to the $0^{\circ}$ slant case, the drag increment for a $30^{\circ}$ slant angle is around $50 \%$ (Grandemange et al. 2013; Lienhart et al. 2002). After this critical angle of $30^{\circ}$, which has the highest drag, a further increase in the slant angle drastically reduces the pressure drag. For example, in Ahmed's body with a $35^{\circ}$ slant angle, the counter-rotating pair of vortices are weak, and the separation occupies the entire slant surface without reattachment at the rear end. Figure 1 illustrates the above point by showing the various regions of physical significance over the 


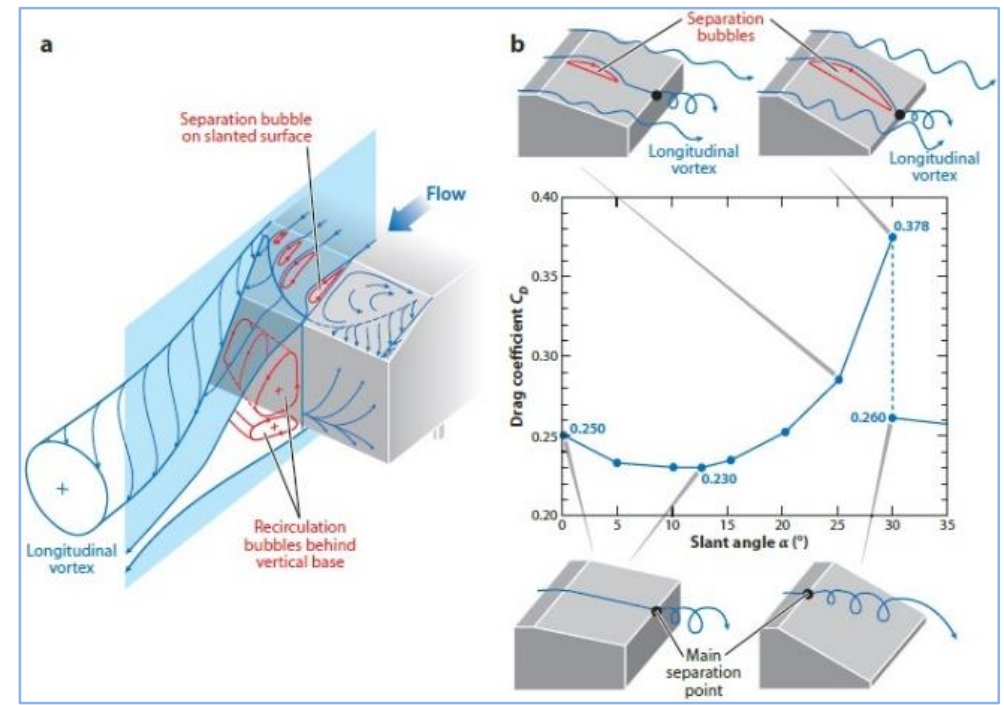

Fig. 1. (a) Time-averaged three-dimensional flow structures of the Ahmed body in the wake and (b) the variation in drag coefficient for different slant angles (Choi et al. 2014).

slant surface of the Ahmed body (Choi et al. 2014).

Figure 1 illustrates the separation bubbles at the slant surface, a pair of counter-rotating longitudinal vortices from the side edges, and recirculation bubbles near the vertical base. These flow features are known to contribute to drag (Ahmed et al. 1984). Based on the existing studies (Choi et al. 2014) suggested two methods to reduce the pressure drag over the Ahmed body model. One way is to maintain the fully attached flow at the slant surface by suppressing the local separation bubbles. This was achieved by connecting an array of circular cylinders just before the slant surface that resulted in a drag reduction (Pujals et al. 2010). Another method of reducing pressure drag is to weaken the strength of the longitudinal vortices emanating from the edges at the slant surface. The early separation of the flow can weaken the C-vortex formation. Some devices, like flaps and vortex generators, have been developed to test this idea and resulted in drag reductions (Beaudoin and Aider 2008; Pujals et al. 2010). Therefore, an understanding of the flow physics over the slant surface is essential for the development of efficient flow control devices. Several active and passive devices have been developed to reduce the aerodynamic drag of road vehicles (Altaf et al. 2014b; Choi et al. 2008; Griffin and Hall 1991). Recently, (Mukut and Abedin 2019) reviewed active and passive devices applied to road vehicles for drag reduction,.

It shows that compared to the active flow control devices, flaps, which are passive devices, provide better drag reduction. For instance, (Beaudoin and Aider 2008) achieved a $25 \%$ drag reduction over a modified Ahmed body with a $30^{\circ}$ slant angle using a rectangular flap. Similarly, (Fourrié et al. 2011) used a small rectangular plate as a deflector over a $25^{\circ}$ slant Ahmed body and achieved a 9\% drag reduction. Another study by (Tian et al. 2017) investigated the effect of flaps over the $25^{\circ}$ and $35^{\circ}$ slant Ahmed body. They achieved a $21.2 \%$ drag reduction for the $25^{\circ}$ Ahmed model and only $6 \%$ drag reduction for the $35^{\circ}$ Ahmed model. A square back long haul MAN TGX truck was simulated by (Altaf et al. 2014a) with three different flap shapes, rectangular, elliptical, and triangular. The elliptical flap obtains a maximum of $11.1 \%$ drag reduction, and the rest were below $6 \%$. Another study on a pickup truck model was conducted by (Ha et al. 2011) using a flap and achieved a $5.6 \%$ drag reduction at a particular size and angle of the flap. Inspired by the secondary feather of birds, a new automatic moving deflector was applied at the $25^{\circ}$ slant Ahmed body model by (Kim et al. 2016). A maximum drag reduction of $19 \%$ was reported when the flap was allowed to vibrate automatically with the incoming flow.

On the other hand, recently, some studies highlighted the physciss of recirculation region and found its relation with the base drag. (Mariotti et al. 2015; Mariotti and Buresti 2013) have established the connection between boundary layer thickness, base drag and the recirculation wake length for an axisymmetric bluff body. An increased boundary layer thickness reduces the base pressure, which extends the mean recirculation bubble. The cause of this extension is attributed to the downstream movement of incipient instability inside the detaching shear layer. However, they cautioned to take this relationship as a cause-effect, as they only found the correlation in all the cases. Similarly, (Barros et al. 2016) investigated a blunt nonaxisymmetric body and found a similar relationship while using high-frequency pulsed jets as an active device. This mechanism reduced the velocity fluctuations at the entire wake, causing shortening of wake entrainment leading to increased recirculation length. Also, flow deviation due to the Coanda effect was found to reduce the wake width. The increased recirculation length and reduced width of the wake collectively resulted in a base drag reduction by $10 \%$. It suggests, again, that base pressure is an increasing 
N. A. Siddiqui and M. A. chaab / JAFM, Vol. 14, No. 1, pp. 147-164, 2021.

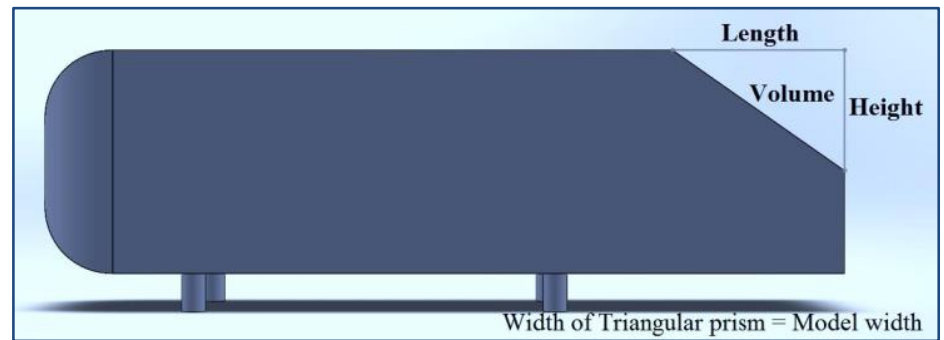

Fig. $2.35^{\circ}$ slant Ahmed body with schematic volume at the slant section.

function of the length of the recirculation length downstream of the body. Moreover, the effect of modifying the spanwise wake or the aspect ratio is also confirmed by the (Mariotti et al. 2019) reporting a $9.7 \%$ base drag reduction. When grooves are applied on a 2D boat-tail, it delays the separation leading to a narrower wake confirming a previous study by ( Mariotti et al. 2017) . By implementing horizontal and vertical deflectors on the square back car, (Capone and Romano 2019) reported a drag reduction caused due to reduced wake in the vertical direction. The modification of the circular vortex at the base as a means of drag reduction is also highlighted by the (Pavia et al. 2019). Therefore, the collective proposal of these investigations strongly suggests that the length, width and height of the recirculation regions is a function of base drag; hence, the manipulation of the recirculation region should be considered a significant criterion to invent and optimize flow control devices. One of the first simulations over the Ahmed body was performed by (Han 1989). He successfully used incompressible turbulence models and reported that $\mathrm{k}-\varepsilon_{\text {, }}$ turbulence model under-predict the base pressure. (Makowski and Kim 2000) used Reynolds-Averaged NavierStoked (RANS) equations to simulate Ahmed body and analyzed the effect of the turbulence model, mesh, and flow structures over the Ahmed body in detail. (Emmanuel Guilmineau 2008) reported that the RANS turbulence models accurately predict the flow behaviour around the $35^{\circ}$ Ahmed body because the flow separation is comparably less robust than the $25^{\circ}$ Ahmed body. Since the $35^{\circ}$ slanted Ahmed body model generates two-dimensional flow behaviour, the prediction about the flow behaviours is accurate. On the contrary, the $25^{\circ}$ Ahmed model creates three-dimensional flow characteristics, which was very well predicted by the turbulence models at the start of a slant. However, turbulence models were not able to show reattachment at the rear end of the $25^{\circ}$ slanted Ahmed body (Emmanuel Guilmineau 2008). Hence, as per (Emmanuel Guilmineau 2008) the $25^{\circ}$ slanted model is a challenge for simulation, but all the other turbulence models, including SST k-omega, capture the flow predictions very well around the $35^{\circ}$ slant Ahmed body. However, (Igali et al. 2019) argued that by precise meshing and descent selection of schemes of discretization, RANS models can anticipate complex three-dimensional flow over the Ahmed body. They proved it using RANS with different turbulence models and found that SST k-omega achieved the best results. Also, (Tian et al. 2017) made excellent results over the Ahmed body using RANS with the SST-k-omega turbulence model.. Recently (Zhang et al. 2019) suggested that RANS with a realizable K-epsilon model performed better than the Largeeddy simulation (LES) models. Therefore, they asserted that RANS can provide numerical results with acceptable accuracy at low cost and fast computation timing. Similarly, (Ashton et al. 2016) stated that the RANS model is almost 17 times more time-effective compared to Detached Eddy Simulation (DES). Furthermore, several studies based on Unsteady Reynolds-Averaged NavierStokes equations (URANS) have been done on Ahmed body; however, for external aerodynamics RANS is the most suitable model (Emmanuel Guilmineau et al. 2011; Hinterberger et al. 2004).

It is found in the literature review that passive flap devices have been used on the side edges on the fastback Ahmed body and at the back of a square back truck body. There is no study on the effect of the flap over the back of a fastback Ahmed body as shown in Fig. 2. A schematic volume is shown at the slanted portion, and this volume is called the slant volume. With several experimental and numerical investigations, it is established that one of the significant reasons for the drag over the fastback Ahmed body is the formation of bubbles inside this slant volume. The bubbles inside the slant volume drastically reduce the pressure, which ultimately increases the overall drag force. Therefore, the possibilities of drag reduction by suppressing these bubbles are promising. Hence, to achieve this aim, a simple rectangular flap is proposed to control the slant volume. By controlling the slant volume, the effect on bubble suppression is investigated.

The objective of the paper is to study the effects of the rectangular passive flap on the slant volume of a fastback Ahmed body. By changing the flap angle, the slant volume is controlled to discover the optimum angle for the drag reduction. It is followed by understanding the physics of the flow to disseminate the mechanism of the drag reduction at the optimal flap angle.

The paper is organized as follows. Section 2 talks about the base model and the details of the passive device. The details of the flow solver are discussed in Section 3. Then Section 4 will report the results and discussions along with the study of the drag reduction mechanism. Vortex identification is discussed in section 5, and Finally, the concluding remarks are reported in Section 6. 
N. A. Siddiqui and M. A. chaab / JAFM, Vol. 14, No. 1, pp. 147-164, 2021.

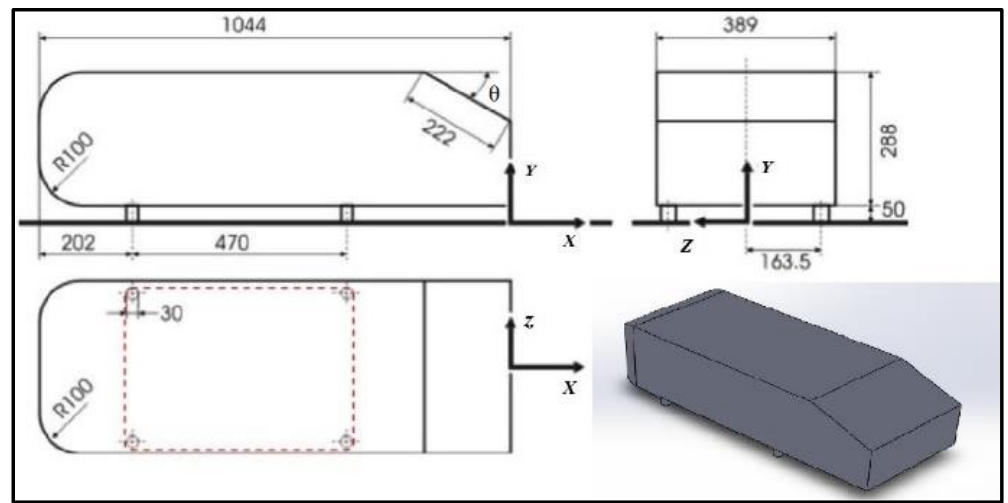

Fig. 3. Dimensions of the Ahmed body (in $\mathrm{mm}$ ), where $\theta=35^{\circ}$.

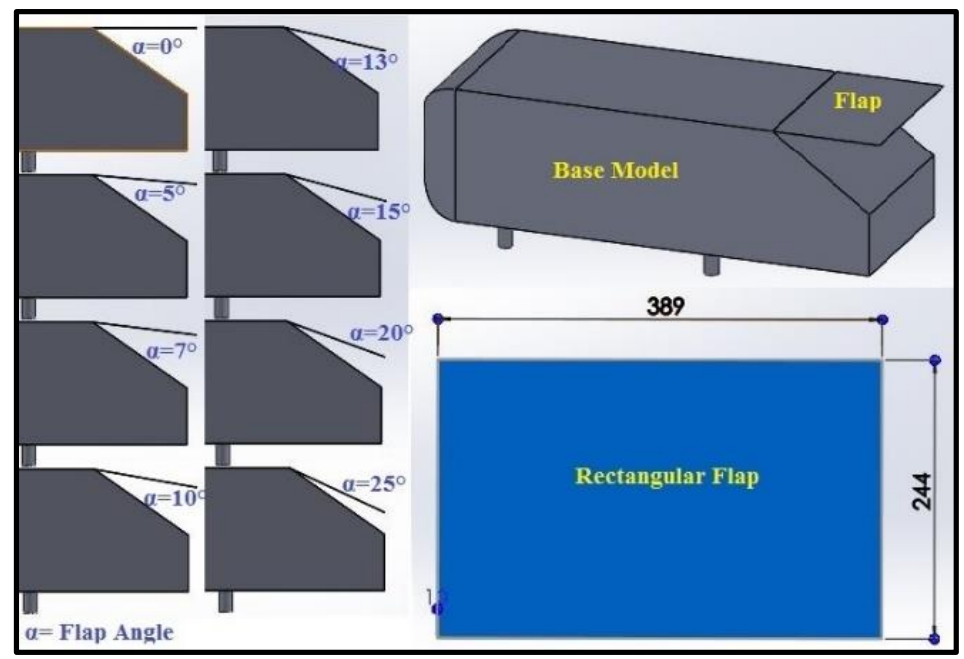

Fig. 4. Ahmed body $35^{\circ}$ slant angle detailed view with Rectangular Flap. The dimensions are in mm.

\section{VEHICle MODEL AND PASSIVE DEVICE DETAILS}

\subsection{Vehicle Model}

As stated earlier, this paper studies the flow around a $35^{\circ}$ slant Ahmed body as a base model. The principal advantage of using this standard vehicle geometry is the availability of the experimental data to validate with the CFD results. Furthermore, in line with the objective of the current investigation, the flow around the $35^{\circ}$ slant surface is well predicted by the RANS with different turbulence models (Guilmineau et al. 2016; Emmanuel Guilmineau 2008). The overall dimensions of the model are shown in Fig.3.

\subsection{Rectangular Flap}

As discussed in Section 1.2, a simple passive device is introduced to control the bubbles generated inside the schematic Ahmed volume shown in Fig.4.

In Fig.4, an isometric view of the base model with the rectangular flap is shown along with the angle $\alpha$. It also shows the dimensions of the rectangular flap where the width is $389 \mathrm{~mm}$, which is equal to the model width, and length is $244 \mathrm{~mm}$. The size of the flap is selected to influence the entire Ahmed volume. On the left side of Fig. 5, all the investigated flap angles are shown. Initially, the flaps were simulated at $5^{\circ}$ steps from $0^{\circ}$ to $25^{\circ}$. Following the results, another set of flap angles of $7^{\circ}$ and $13^{\circ}$ were simulated to assess the effect near the flap angle $10^{\circ}$, which provides the maximum drag reduction.

\section{SIMULATION DETAILS}

\subsection{Mathematical Model}

The numerical simulation was performed using commercially available software, Ansys Fluent, which is based on the Finite Volume Method. The governing equations for the steady-state flow conditions are used to calculate the flow around the Ahmed body. Through Reynolds decomposition, the instantaneous Navier-Stokes equations are converted into Reynolds-Averaged Navier-Stokes equations for incompressible turbulent flow. The steady-state RANS equations for mass and momentum are:

\section{Continuity}

$\frac{\partial}{\partial \mathrm{x}_{\mathrm{i}}}\left(\overline{\rho \overline{\mathrm{u}}_{1}}\right)=0$

Momentum 


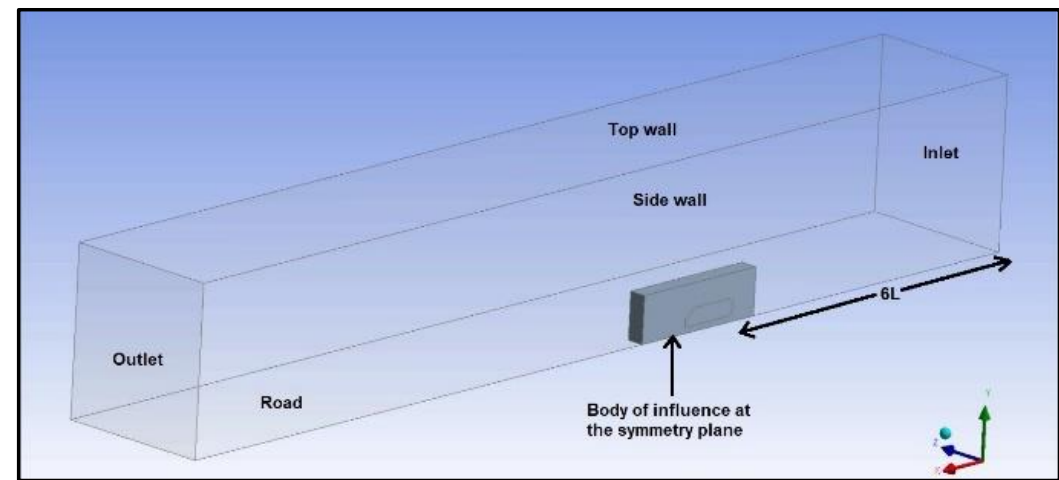

Fig. 5. Computational domains with key dimensions and boundary conditions.

$$
\begin{aligned}
& \frac{\partial}{\partial \mathrm{x}_{\mathrm{j}}}\left(\overline{\rho \mathrm{u}_{\mathrm{l}} \mathrm{u}_{\mathrm{j}}}\right)=-\frac{\partial \overline{\mathrm{p}}}{\partial \mathrm{x}_{\mathrm{i}}}+\frac{\partial}{\partial \mathrm{x}_{\mathrm{j}}}\left[\mu \left(\frac{\partial \overline{\mathrm{u}}_{1}}{\partial \mathrm{x}_{\mathrm{j}}}+\frac{\partial \overline{\mathrm{u}}_{\mathrm{j}}}{\partial \mathrm{x}_{\mathrm{i}}}-\right.\right. \\
& \left.\left.\frac{2}{3} \partial_{\mathrm{ij}} \frac{\partial \overline{\mathrm{u}}_{1}}{\partial \mathrm{x}_{1}}\right)\right]+\frac{\partial}{\partial \mathrm{x}_{\mathrm{j}}}\left(-\rho \overline{\mathrm{u}_{1}^{\prime} \mathrm{u}_{\mathrm{j}}^{\prime}}\right)
\end{aligned}
$$

Here $\bar{\rho}$ is the mean density, $\bar{p}$ is the mean pressure, $\mu$ the molecular viscosity and $-\rho \overline{u_{\imath}^{\prime} u_{\jmath}^{\prime}}$ are the Reynolds stresses. These equations represent the solution variables in an averaged form. The additional closure terms are on the right side of the Eq. (2), that need to be modelled to close the solution. As stated earlier, the Shear stress transport k-omega model is used to close Eq. (2). The term $-\rho \overline{u_{\imath}^{\prime} u_{\jmath}^{\prime}}$ Constitutes a Reynolds tensor that gives a total of nine components. However, out of nine, only six are independent variables, and the solution of these unknowns can be obtained analytically. This problem is known as the closure problem, and it is resolved by applying turbulence modelling.

\subsection{Turbulence Modeling}

The SST turbulence model consists of the $k-\omega$ and $k-\varepsilon$ model models. The equations of models are as follows as per the (Igali et al. 2019; Menter 1994):

$$
\begin{aligned}
& \frac{\partial(\rho k)}{\partial t}+\frac{\partial\left(\rho u_{j} k\right)}{\partial x_{j}} \\
&=\rho P-\beta \rho \omega k+\frac{\partial\left(\left(\mu+\sigma_{k} \mu_{t}\right) \frac{\partial k}{\partial x_{j}}\right)}{\partial x_{j}} \\
& \frac{\partial(\rho \omega)}{\partial t}+\frac{\partial\left(\rho u_{j} \omega\right)}{\partial x_{j}}=\frac{\gamma}{v_{t}} P-\beta \rho \omega^{2} \\
&+\frac{\partial\left(\left(\mu+\sigma_{\omega} \mu_{t}\right) \frac{\partial \omega}{\partial x_{j}}\right)}{\partial x_{j}} \\
&+2(1 \\
&\left.-F_{1}\right) \frac{\rho \sigma_{\omega 2}}{\omega} \frac{\partial k}{\partial x_{j}} \frac{\partial \omega}{\partial x_{j}} \\
& P=\tau_{i j} \frac{\partial u_{i}}{\partial x_{j}}
\end{aligned}
$$

The constant $\emptyset$ of the model is calculated by:

$\emptyset=F_{1} \emptyset_{1}+(1-F) \emptyset_{2}$

Eddy Viscosity

$$
v_{t}=\frac{\alpha_{1} k}{\max \left(\alpha_{1} \Omega ; \Omega F_{2}\right)}
$$

The $\Omega$ is the absolute value of the vorticity:

$$
\begin{gathered}
F_{1}=\tanh \left(\operatorname{ar} g_{1}\right) F_{1}=\tanh \left(\operatorname{ar} g_{2}\right) \\
\operatorname{ar} g_{1}=\min \left[\max \left(\frac{\sqrt{k}}{0.09 \omega y} ; \frac{500 v}{y^{2} \omega}\right), \frac{3.424 p k}{C D_{k \omega} y^{2}}\right] \\
\operatorname{ar} g_{2}=\max \left(2 \frac{\sqrt{k}}{0.09 \omega y} ; \frac{500 v}{y^{2} \omega}\right) \\
C D_{k \omega}=\max \left(1.712 \rho \frac{1}{\omega} \frac{\partial k}{\partial k_{j}} \frac{\partial \omega}{\partial x_{j}}, 10^{-20}\right.
\end{gathered}
$$

\subsection{Domain and the Boundary Conditions}

The extent of the flow domain is $15 \mathrm{~L} \times 6 \mathrm{~L} \times 3 \mathrm{~L}$ (where $\mathrm{L}=$ length of the model) shown in Fig. 5. To improve the quality of the mesh, a resolution box is created around the model (also called body of influence) with dimensions of $0.5 \mathrm{~L}$ at the front, $1 \mathrm{~L}$ at the backside, and $0.5 \mathrm{~L}$ in height, as shown in Fig. 5. (Lanfrit 2005).

The freestream velocity is set to the inlet as $40 \mathrm{~m} / \mathrm{s}$, leading to a Reynolds number of $7.8 \times 10^{5}$ based on model height. The road is a non-moving surface over which the model is placed $50 \mathrm{~mm}$ above as per the experimental setup of (Ahmed et al. 1984).

\subsection{Meshing}

Since the model is geometrically symmetrical, symmetry was applied in the simulation. After a gridindependent test with five different sizes, a fine mesh with more than 6 million elements used. Prism layers are used to capture the boundary layer flow. A total of 5 inflation layers are used at the boundary layer with a growth rate of $1.2 \%$, which is in line with the best practice guideline given by Fluent for the external aerodynamics (Lanfrit 2005). For the meshing, triangular, and tetrahedral elements are used. The $\mathrm{Y}^{+}$values around the model are in the range of 30 to 150 . Moreover, near the wall, this value is below 6 .

Figure 6 shows mesh around the model. The mesh at the symmetry plane is shown in Fig. 6 (a), and the corresponding side-view captures the prism layer growth in (b). The inflation boundary layer is also 
depicted in (c), and a complete section view is shown in (d).

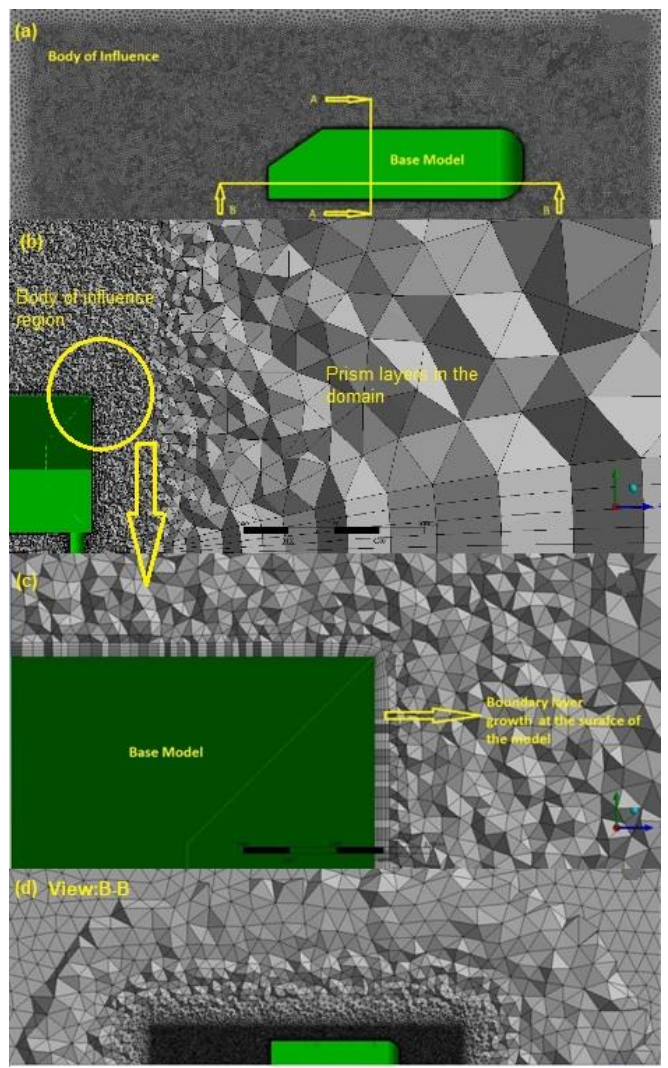

Fig. 6. Meshing around the base model with prism layer development: (a) Base model with the body of influence, (b) Enlarged view of the slant area, and (c) Boundary layer growth.

Table 1 Drag coefficient validation with the

\begin{tabular}{|c|c|c|c|c|}
\hline $\begin{array}{c}\text { Sr. } \\
\text { No. }\end{array}$ & Reference & Method & $\begin{array}{c}\text { Drag } \\
\text { coefficient }\end{array}$ & $\begin{array}{c}\% \\
\text { Accuracy }\end{array}$ \\
\hline $\mathbf{1}$ & $\begin{array}{c}\text { (Ahmed } \text { et } \\
\text { al. } 1984)\end{array}$ & Experiment & 0.260 & Base \\
\hline $\mathbf{2}$ & $\begin{array}{c}\text { (Tian } \text { et al. } \\
\text { 2017) }\end{array}$ & $\begin{array}{c}\text { CFD } \\
\text { Simulation }\end{array}$ & 0.292 & 11 \\
\hline $\mathbf{3}$ & $\begin{array}{c}\text { (Emmanuel } \\
\text { Guilmineau, } \\
\text { 2008) }\end{array}$ & $\begin{array}{c}\text { CFD } \\
\text { Simulation }\end{array}$ & 0.313 & 20 \\
\hline $\mathbf{4}$ & $\begin{array}{c}\text { Present } \\
\text { study }\end{array}$ & $\begin{array}{c}\text { CFD } \\
\text { Simulation }\end{array}$ & 0.271 & 4 \\
\hline
\end{tabular}

\subsection{CFD Validation}

The drag coefficient obtained in this study is compared with the available data in the literature in Table 1. The drag coefficient obtained in the present study is in agreement with the experimental value (Ahmed et al. 1984). The represent result is also consistent with previous studies, as shown in the table. Also, the fractional bias (FB) of the present study $\left(2 \frac{\overline{\left(\mathrm{u}_{\mathrm{s}}+\mathrm{u}_{\mathrm{e}}\right)}}{\overline{\mathrm{u}_{\mathrm{s}}+\overline{\mathrm{u}_{\mathrm{e}}}}}\right)$ is 0.2 considering the experimental values of (Ahmed et al. 1984), which is in the acceptable range of $-2 \leq \mathrm{FB} \leq+2$ recommended by (Emery et al. 2017). Where $u_{s}$ is simulation velocity, and $u_{e}$ is the experimental value.

\subsection{Results and Discussions}

\subsection{Drag Force}

As the pressure drag dominates a bluff body, the drag force is obtained from the well-known drag equation as follow:

$$
\mathrm{C}_{\mathrm{D}}=\frac{\mathrm{F}_{\mathrm{D}}}{1 / 2 \rho \mathrm{Uref}^{2} \mathrm{~A}}
$$

Where $\boldsymbol{F}_{\boldsymbol{D}}$ is the drag force, $\boldsymbol{\rho}$ is density, $\mathrm{U}_{\text {ref }}$ the freestream velocity, and A is the projected area of the body. The variation of the drag coefficient with the flap angle is shown in Fig 7.

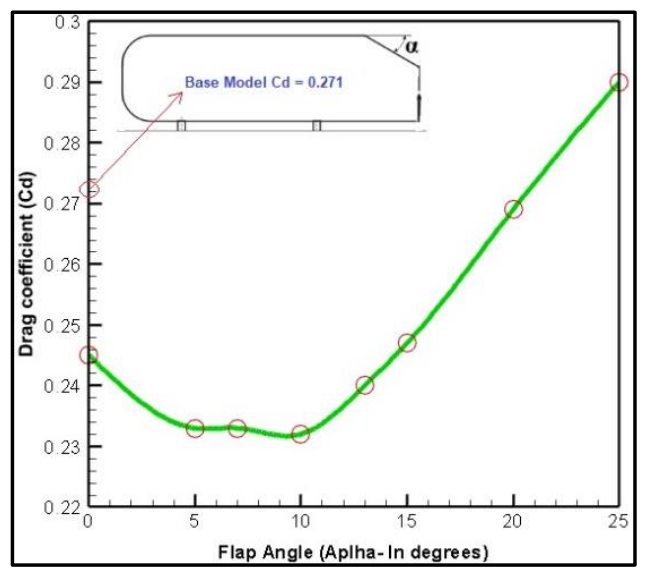

Fig. 7. Drag coefficient variation with flap angle.

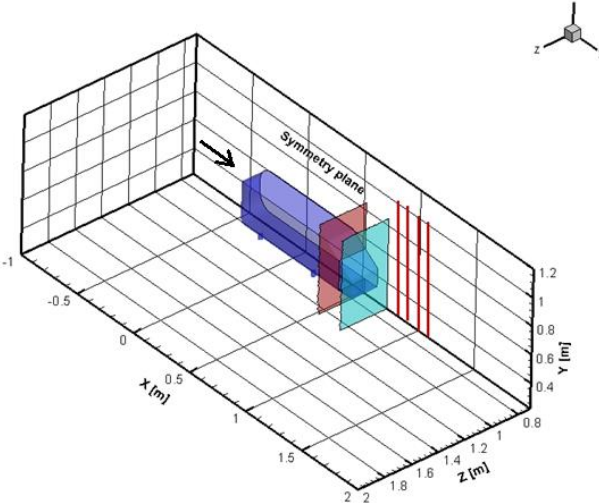

Fig. 8. Plane locations for the investigation. In the $X$-direction, $X=0.67 \mathrm{~m}$ and $X=0.87 \mathrm{~m}$. In the $Z$-direction symmetry plane, which is at $Z=$ $0.8438 \mathrm{~m}$ and second plane at $\mathrm{Z}=1.03 \mathrm{~m}$ to capture the effect of induced drag. The red line are at position $X=0.8,0.9,1.0$ and $1.3 \mathrm{~m}$. the kinetic energy data is extracted along the red lines for the comparison in subsequent sections. The arrow shows the wind direction.

In the first phase of the investigation flap angle in a range of $0^{\circ}, 5^{\circ}, 10^{\circ}, 15^{\circ}, 20^{\circ}$, and $25^{\circ}$ are simulated It is clear from Fig. 8 that with the addition of a 


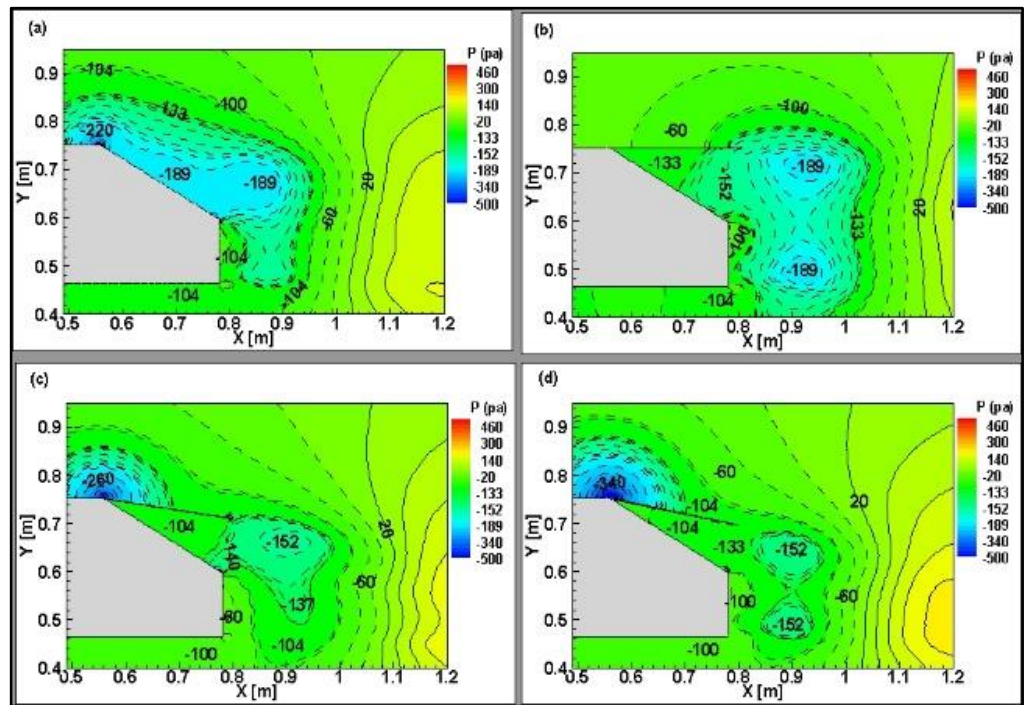

Fig. 9. Pressure (pa) contours at the plane $Z=0.8438 \mathrm{~m}$ (a) Base model, and with the flap at (b) $\alpha=0^{\circ}$ (c) $\alpha=10^{\circ}$ (d) $\alpha=13^{\circ}$. (Dashed lines represent the negatives values of the pressure).

simple rectangular flap at an angle to the slant surface has a considerable drag reduction. When the rectangular flap is at a zero degree angle, it provides a drag reduction of $9.5 \%$ compared to the base model. This drag reduction continually increases to a maximum of $14.3 \%$ at $10^{\circ}$. Beyond this it starts to increase.

As stated earlier, $7^{\circ}$ and $13^{\circ}$ flap angles were further investigated to ascertain that $10^{\circ}$ indeed has the maximum drag reduction. The results confirmed that the $\mathrm{Cd}$ values for $7^{\circ}$ and $13^{\circ}$ are higher than the $10^{\circ}$ case.

\subsection{Flow Field Analysis}

The flow field analysis is divided into two parts. In the first part, the optimal $10^{\circ}$ case is compared with the base model and along with the $0^{\circ}$ and $13^{\circ}$ flap angles using pressure, velocity data. Since the drag coefficients at $5^{\circ}$ and $7^{\circ}$ are close to the $10^{\circ}$ case, the $0^{\circ}$ flap is compared instead. Then in the second part, a detailed comparison of the base model and the optimal angle $10^{\circ}$ case is performed. The goal here is to provide an overall and specific flow features of the flap.F low variables are investigated along the planes and lines shown in Fig. 8 .

\subsection{Pressure Analysis}

Drag reduction depends on the pressure recovery at the rear end of a bluff body. Figures 9 and 10 demonstrate the pressure field of the compared models at the symmetry plane, and the second plane is taken near the side edges of the Ahmed body to capture the effect of the longitudinal vortex at $\mathrm{Z}=$ $1.03 \mathrm{~m}$, respectively.

At the symmetry plane, the pressure recovery at the slant surface is apparent for all the cases compared to the base model, which has a pressure of -189 . The flap with a $0^{\circ}$ angle recovered $29.6 \%$ (with $-133 \mathrm{~Pa}$ ) of pressure compared to the base model. The $10^{\circ}$ degree angle provides a pressure resurgence of
$44.9 \%$ (with -104 pa). Moreover, $13^{\circ}$ flap angle also shows a $44.9 \%$ (with -104 pa). However, the achieved drag coefficient for the $10^{\circ}$ case is 0.232 , and the $13^{\circ}$ angle raises it to 0.240 . This boost is since recirculation bubbles formed behind the base of the model indicate a higher retrieval of pressure in the $10^{\circ}$ case with -60 pa compared to the -100 pa for the $13^{\circ}$ flap. Therefore the total pressure resurgence at the $10^{\circ}$ case is $87.2 \%$ compared to the base model. Hence, a $10^{\circ}$ angle provides the optimal angle of drag reduction.

As mentioned above, sources of drag on the Ahmed model include slant surface separation bubbles, recirculation bubbles at the vertical base and longitudinal vortex from the side edges. The slant surface separation bubbles are evident in the base model (i.e., Fig. 9a) and occupy the entire slant area. Moreover, the bubbles extend outside the slant surface ending before the $X=0.9 \mathrm{~m}$. Such separation bubbles cause a vast negative pressure region to increase the pressure drag. The reason for these large bubbles is the fixed separation point at the start of the slant surface.

However, at the $0^{\circ}$ flap angle (Fig. $9 \mathrm{~b}$ ), the length of the flap delays the separation point. The delay in separation creates reflectional symmetric bubbles at the end of the flap. The flap transfers the slant surface bubble to the end of the flap with almost identical pressure of $-189 \mathrm{pa}$. This shift is almost $160 \mathrm{~mm}$ away from the model base and provides space for pressure recovery between the flap and slanted surface. Though, the pressure recovery at the slant volume is not entirely the same because the existence of two different negative pressure zones (-133pa and $152 \mathrm{pa})$ is evident. These two zones are the direct exposure to the symmetric bubbles that influence the slant surface.

Nevertheless, for the $10^{\circ}$ flap (Fig. 9c), the symmetrical structure of the bubbles breaks down, and the separation point is modified according to the 


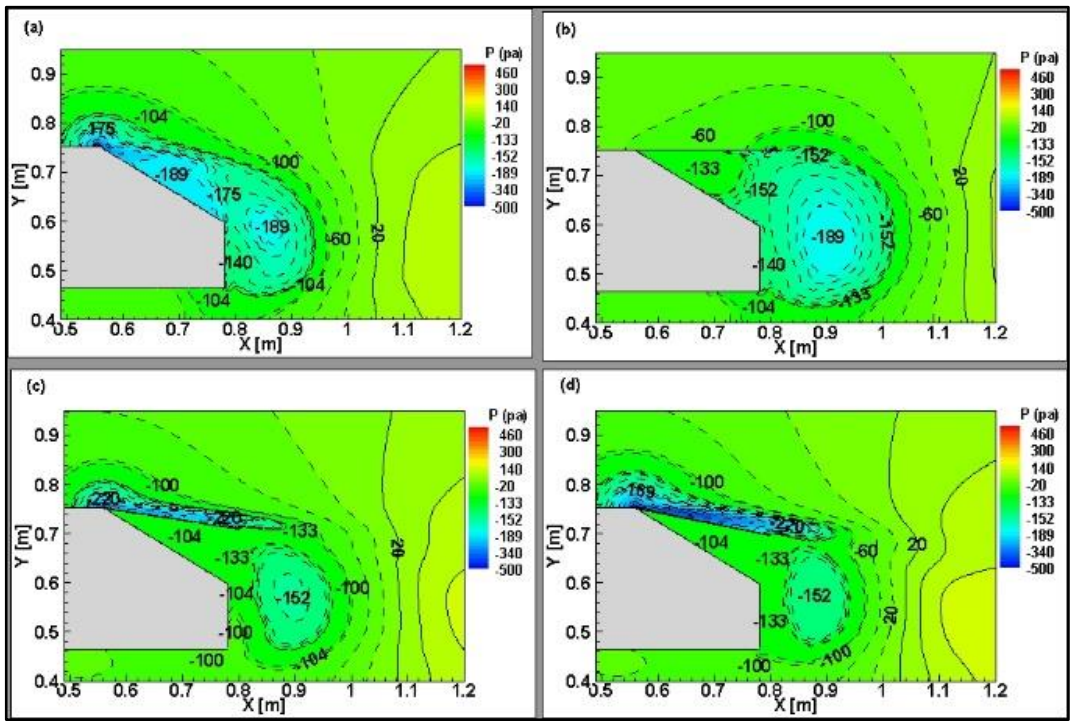

Fig. 10. Pressure (pa) contours at the $Z=1.03$ (a) Base model (b) $\alpha=0^{\circ}$ (c) $\alpha=10^{\circ}$ (d) $\alpha=13^{\circ}$. (Dashed lines represent the negatives pressure).

flap angle. This symmetry breaking transforms into a circular shape bubble that has a lower negative pressure (-150 pa). The separation bubble now rests at $100 \mathrm{~mm}$ from the vertical base, which is less than the $0^{\circ}$ case. The reduced distance affects the pressure at the slant volume near the flap end. However, almost the entire slant volume is occupied by the negative pressure of $-104 \mathrm{pa}$ causing significant pressure recovery. Moreover, a rather small circular bubble compared to the symmetric in the $0^{\circ}$ case allows further pressure recovery at the vertical base with a pressure of $-60 \mathrm{pa}$. Therefore, the exposure of modified separation bubbles influences a rather small region, which allows pressure insurgence at the slant volume and vertical base.

The importance of symmetry breaking is further evident in the $13^{\circ}$ flap case. The existence of small symmetric bubbles is apparent in Fig. 9d. Hence, the effect of slant volume becomes more transparent, which is a function of the flap angle. As discussed above, the $0^{\circ}$ case demonstrates quite a large symmetric bubbles due to delay in separation as the slant volume is reduced with the flap angle $10^{\circ}$, the symmetric bubbles breaks-down and transformed into a small circular bubble at the end of the flap. Interestingly, a further decrease in the slant volume with a $13^{\circ}$ flap angle recreates the reflectional symmetric bubble though comparatively small but with -152pa pressure. The upper half of the recreated symmetric bubble influence the slant volume at the end of the flap with a pressure of -133pa. Nonetheless, at this location, it is similar to the $10^{\circ}$ case whereby the lower half portion faces the vertical base and increases the pressure to -100pa. Therefore, the difference lies at the vertical base where a $10^{\circ}$ case allows a pressure recovery, but a $13^{\circ}$ case increases the pressure due to recreated symmetric bubbles.

The pressure analysis at the symmetry plane reveals the pressure recovery at the slant volume correlation with the reflectional symmetry breaking Nonetheless, it does not capture the effect of the third important drag source in the Ahmed body, which is longitudinal vortex coming out of side edges. Hence, Fig. 10 captures the pressure field near the side edge of the body at the plane $\mathrm{Z}=1.03 \mathrm{~m}$.

As for the base case (Fig. 10a), the separation bubbles still exist at the slant surface with -189 pa pressure, but compared to the symmetry plane, it does not occupy the whole slant surface. At the vertical base, the pressure is increased to -140pa. It is due to the changes in the separation bubbles at the slant volume.

At the $0^{\circ}$ flap angle, there exists a large separation due to a delay in the separation. It contrasts the symmetric bubble available at the symmetry plane. The bubble shows the same pressure values as the base model, which is -189 pa at the core. As for the outer side of this large bubble, pressure drops up to $140 \mathrm{pa}$. The center of this bubble is around $120 \mathrm{~mm}$ far from the vertical base, which allows a pressure recovery inside the slant volume similar to the symmetry plane. Figure $10 \mathrm{~b}$ also demonstrates that there is no sign of the longitudinal vortex. It is corroborated by the iso-surface of the pressure for the $0^{\circ}$ shown in Fig. $11 \mathrm{~b}$. Iso-surface is formed at the end of the flap without showing the existence of a longitudinal vortex.

Furthermore, with a reduction in slant volume at a $10^{\circ}$ angle, the separation bubble segregates from the flap and forms a comparatively small circular bubble. The core of this bubble has a pressure of $152 \mathrm{pa}$ and is away around $120 \mathrm{~mm}$ from the vertical base. Compared to the symmetry plane (Fig. 9c), here does not influence the slant volume, hence allowing pressure recovery with the pressure value of -104 pa. Additionally, it reduced the vertical base pressure from -140pa to -100pa compared to the base and $0^{\circ}$ case. However, above the flap, there is a layer of high negative pressure of -220pa. It begins with 


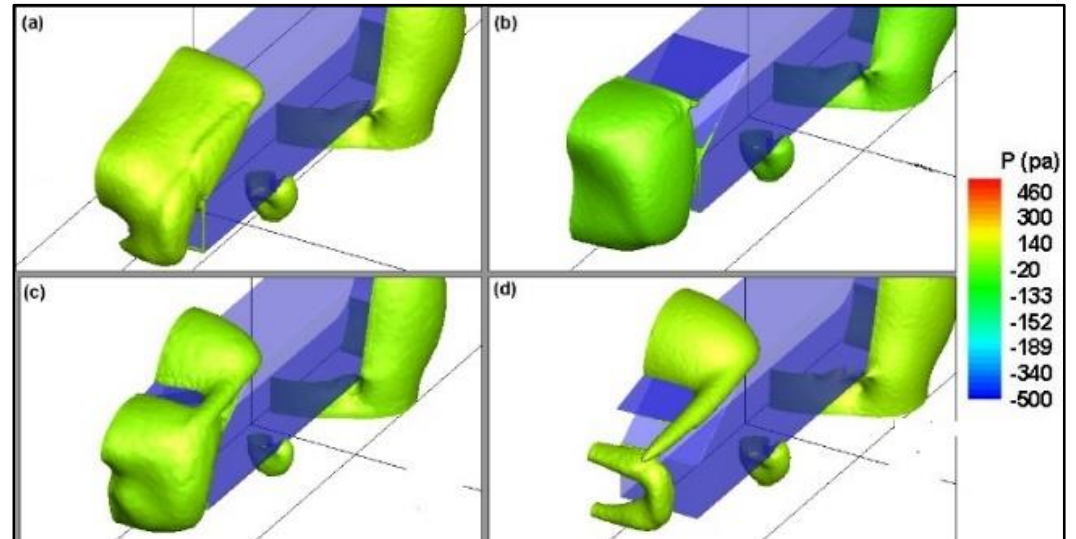

Fig. 11. Iso-surface of the pressure (pa) at the Iso-value of -150 (a) Base model (b) $\alpha=0^{\circ}$ (c) $\alpha=10^{\circ}$ (d) $\alpha=13^{\circ}$. The iso-value of -150 is chosen to capture the best possible pressure Iso-surfaces.

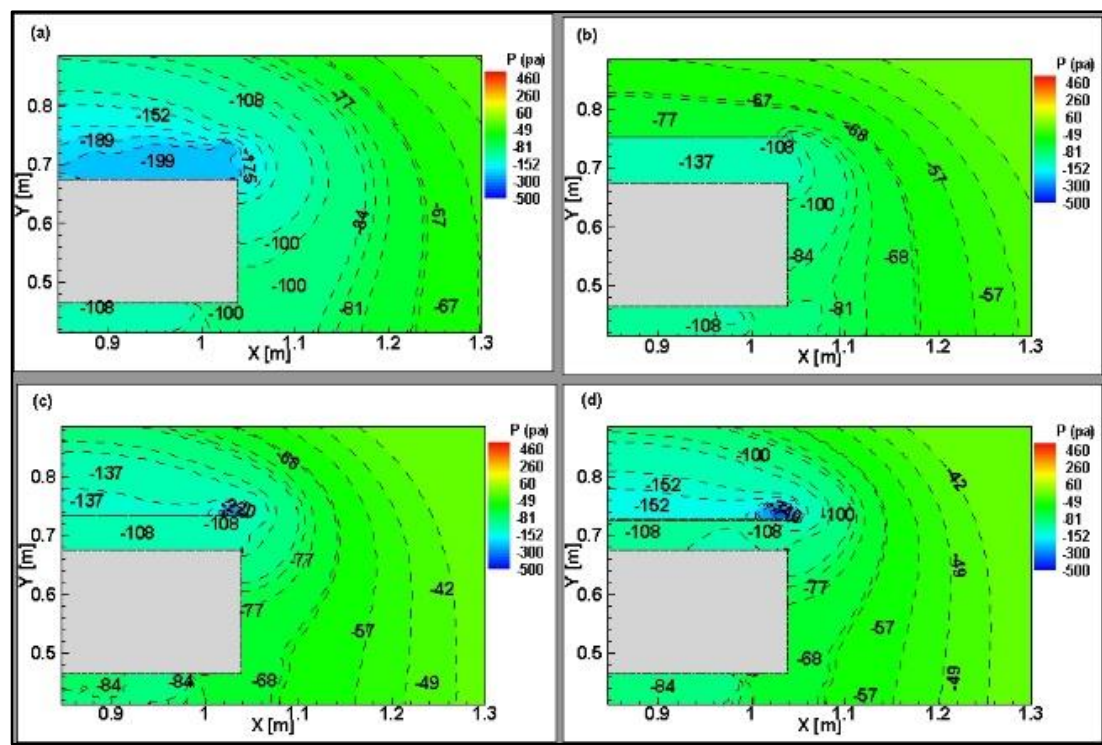

Fig. 12. Pressure (pa) contours at the $X=0.67 \mathrm{~m}$ (a) Base model (b) $\alpha=0^{\circ}$ (c) $\alpha=10^{\circ}$ (d) $\alpha=13^{\circ}$ (Dashed lines represent the negatives values of the pressure).

the start of the slant and extends the entire flap length, which demonstrates the existence of a longitudinal vortex at the side edges. It is confirmed from the iso-surface of the pressure shown in Fig. $11 \mathrm{c}$, which captures the longitudinal vortex. Nonetheless, it does not contribute to the pressure recovery.

A further reduction in slant volume reduced the size of separation bubbles and positioned it to $100 \mathrm{~mm}$ from the vertical base at pressure -152pa. Apart from the higher pressure at the vertical base of $-133 \mathrm{pa}$ compared to the $10^{\circ}$ case, the pressure recovery at the slant volume remains the same. Moreover, above the flap, a layer of very high negative pressure of $324 \mathrm{pa}$ exists, capturing the longitudinal vortex at the side edges. The existence of the side vortex is reflected in Fig. 11d showing iso-surface of the pressure. It is highly developed in the $13^{\circ}$ case, which suggests that the further increase in the drag coefficient after a $10^{\circ}$ depends on the contribution made by vertical base pressure and the longitudinal vortex.

The cross-sectional pressure distribution at the $\mathrm{X}=$ $0.67 \mathrm{~m}$ (Fig. 12) and $X=0.87 \mathrm{~m}$ (Fig. 13) demonstrates the major differences.

At the slant surface plane ( $\mathrm{X}=0.67 \mathrm{~m}$ ) in Figs. 9, a high-pressure recovery is evident. The effect of slant volume is clear, and compared to the base model, reduced pressure values are reported in all the flap cases. At both the planes, the existent of a longitudinal vortex is visible for the $10^{\circ}$ and $13^{\circ}$ cases. Although the pressure inside the $10^{\circ}$ longitudinal vortex is $220 \mathrm{pa}$, however, it increased to $-324 \mathrm{pa}$ for the $13^{\circ}$ case. Hence, the augmenting longitudinal vortex abates the pressure, which amplifies the drag. It is the major divergence from the $10^{\circ}$ case. Moreover, plane $\mathrm{X}=0.87$ captures the pressure at the bubble formation area. It re-stresses the pressure difference available at the core of bubbles for all the cases. Yet, the minimum is recorded as $-122 \mathrm{pa}$ around the symmetry plane in a $10^{\circ}$ case. 


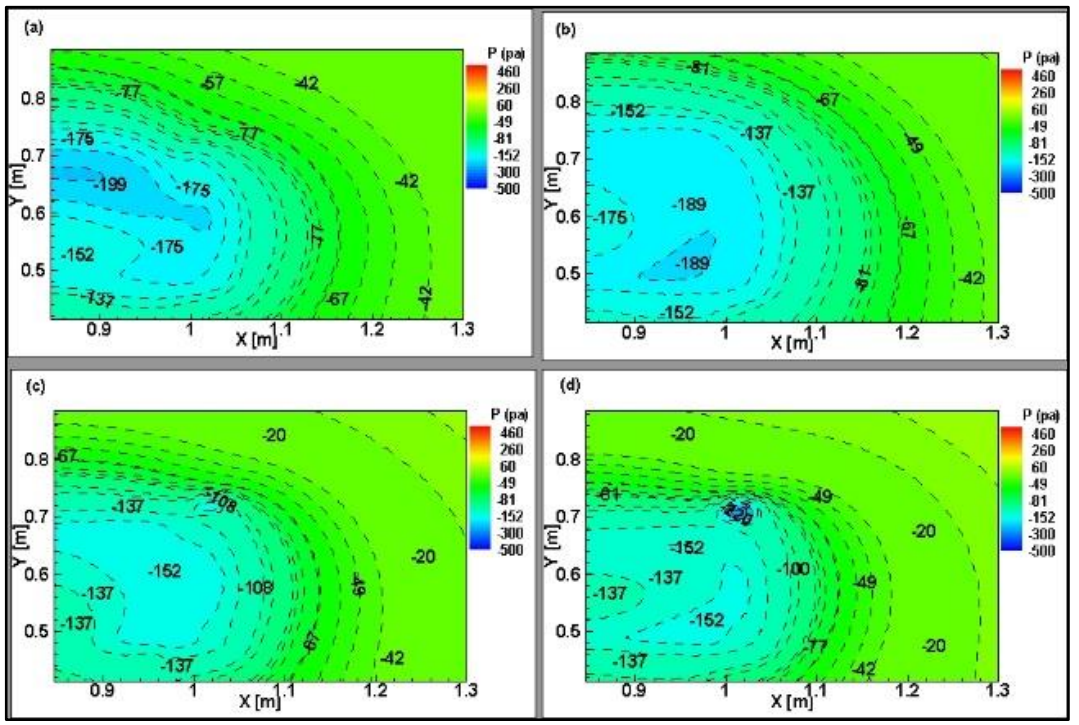

Fig. 13. Pressure (pa) contours at the $X=0.87 \mathrm{~m}$ (a) Base model (b) $\alpha=0^{\circ}$ (c) $\alpha=10^{\circ}$ (d) $\alpha=13^{\circ}$ (Dashed lines represent the negatives values of the pressure).

Additionally, Fig. 13d illustrates the longitudinal vortex formed from the side edges, which is more developed than the $10^{\circ}$ case with -228 pa pressure at the core.

The results revealed the effect of controlled slant volume on the drag reduction. As the flap angle begins to increase from $0^{\circ}$, it reduces the slant volume until the flap angle of $10^{\circ}$ is reached. The decrease in slant volume brings about a reduction in the drag force. However, a further decrease in the slant volume increases the drag force. The primary reason for pressure recovery is realized due to the suppression of the separation bubbles at slant volume. Due to the delay in separation caused by the added flap, the separation bubbles shift from slant volume to the end of the flap. It provides a gap at the slant volume for pressure recovery. Furthermore, a minor contribution is made by the destruction of the recirculation bubbles near the vertical base. That makes the $10^{\circ}$ angle crucial for the physical understanding of the drag reduction.

From Fig. 7, it is clear that after $10^{\circ}$, there is a linear relationship between the drag coefficient and the flap angle. Hence, the $10^{\circ}$ provides the minima of the drag reduction due to the flap. Therefore, the slant volume formed due to the $10^{\circ}$ angle is the most optimal volume to suppress the bubbles.

\subsection{Velocity Analysis}

Ahmed body has a fixed separation point followed by a recirculation region of adverse pressure gradients (Choi et al. 2014; Guilmineau et al. 2016; Igali et al. 2019). The separation of the shear layer produces a strong reverse flow in the downstream wake. This reverse flow is a closed-region with a reattachment line. The relation between base drag and recirculation region's overall aspect ratio has been documented by (Barros et al. 2016; Capone and Romano 2019; Mariotti 2018; Mariotti et al. 2015, 2017, 2019; Alessandro Mariotti and Buresti, 2013;
Pavia et al. 2019) anddiscussed in the introduction.

The above relation is also evident while implementing a simple passive flap on the slanted Ahmed body. Figure 14 shows the zero velocity line that quantifies the extent of the backflow region. With the separation line fixed at the start of the slanted surface, the base case shows a fully developed recirculation region. The backflow region begins from the start of slant volume and extends up to $\mathrm{X}=1.15 \mathrm{~m}$, a backflow region worth $0.56 \mathrm{~m}$, as shown in Fig. 14. In contrast, the backflow region of a $10^{\circ}$ case begins from $0.73 \mathrm{~m}$ inside slant volume and extends up to $\mathrm{X}=1.2 \mathrm{~m}$. It creates a backflow region of $0.47 \mathrm{~m}$, which is an $18.1 \%$ reduction from the base model. The cause of this extended backflow region is the delay in flow separation well documented by (Mariotti et al. 2015, 2019). In the present case, it is due to the flap shifting the separation bubble behind the vertical base. Hence, the first feature of the drag reduction due to extended recirculation length (R.L) is evident for the flap shown in Figs. $14 \& 18$.

The quantification of this backflow region is essential to understand the mechanism of drag reduction (Fernholz and Urzynicok 2006) Therefore, the velocity field is shown in Figs. 15-17. For the base case (Fig. 15a), the dominant velocity at the slant volume is $-5 \mathrm{~m} / \mathrm{s}$, and the $10^{\circ}$ case (Fig. 15b) has a zero velocity. The cross-sectional velocity field at the slanted surfaces is shown in Fig. 16. In the spanwise direction, the slanted surface is filled by the negative velocities (shown in dashed lines, $-5 \mathrm{~m} / \mathrm{s}$ ) in the base case. It means that the whole span of the base slant surface has reverse flow. However, for the $10^{\circ}$ flap, the entire span of the slant volume has a zero velocity. It indicates pressure energy throughout the slant volume. Nonetheless, due to longitudinal vortex, the velocity increased to positive $5 \mathrm{~m} / \mathrm{s}$, providing insight about the comparatively low-pressure region towards the side edge. 


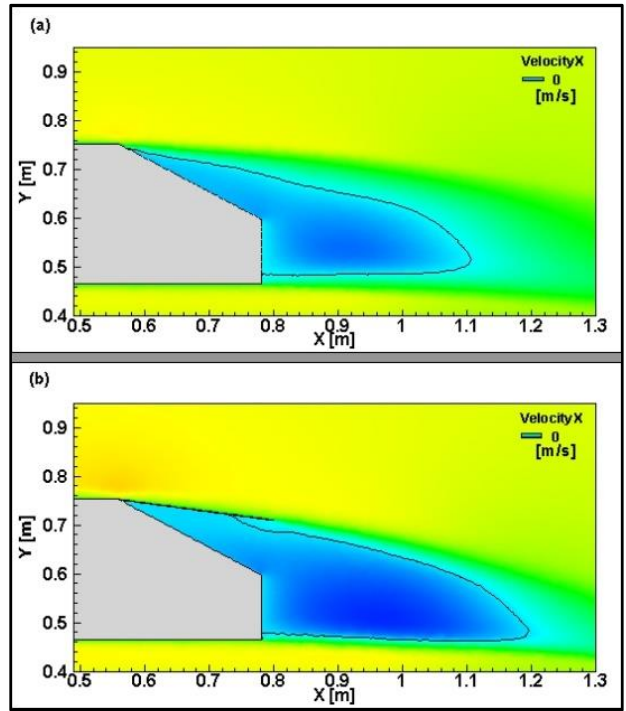

Fig. 14. Zero velocity contour line at the symmetry plane (a) Base model (b) $\alpha=10^{\circ}$.

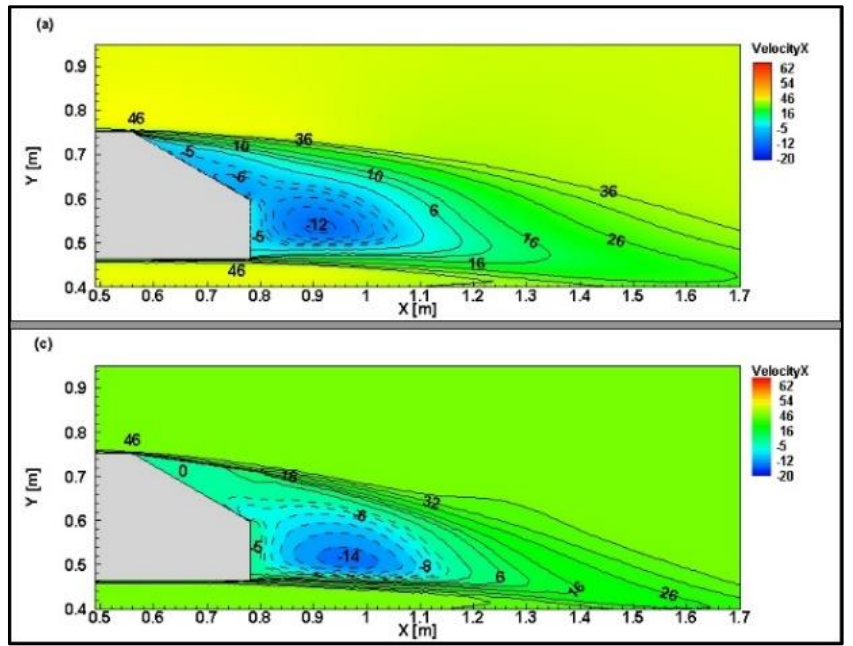

Fig. 15. Velocity $X$ contours at the $Z=0.84 m$ (a) Base model (b) $\alpha=10^{\circ}$ (Dashed lines represent the negatives values of the velocity).

Moreover, in the downstream direction behind the vertical base, the cross-section velocity is shown in the plane $X=0.87 \mathrm{~m}$ Fig. 17 . This location is another region where the reduction in the spanwise backflow region is evident. The backflow region is shown with zero velocity contour lines in Fig. 17. For the base case, the region extends up to $\mathrm{Z}=1.02 \mathrm{~m}$ in contrast to the $10^{\circ}$ case, where it breaks away at $Z=0.95 \mathrm{~m}$. This decrease in the cross-section of the wake causes pressure recovery at the vertical base (Barros et al. 2016; Gerrard 1966; Roshko 1955). It also implies that compared to the base model, the spanwise reduction in the backflow region is higher in the $10^{\circ}$ case, which is clear from the Fig. 17. Therefore, the second feature of the drag reduction due to the reduced recirculation width (R.W) is evident for the flap case in Figs. $17 \&$ 18. This is consistent with Barros et al. 2016.

The third variation in the backflow region proposed by (Capone and Romano 2019) is the reduction in the vertical direction. From Figs. 14 \& 17, the addition of the flap does not influence the recirculation height
(R.H) in the vertical direction. Compared to the base case, it is almost the same. A breakdown of the backflow region is tabulated in Table 2. Within this recirculation region, the fluctuation of kinetic energy is shown in the vortex identification section for further understanding.

The difference in the present study lies in the use of slanted Ahmed body with a simple passive flap device to alter the structure of the flow separation. However, there is similarity in terms of the extended recirculation region with the studies of both ( Mariotti et al. 2015) and (Barros et al. 2016). Also, the similarity in terms of an abridged wake width reported by Barros et al. 2016, which they found was due to the Coanda effect. Mariotti et al. 2015 attributed the elongation to the increased boundary layer thickness before separation. However, Barros et al. 2016 found that at higher actuation frequencies, the turbulent kinetic energy is reduced, which abridged the entrainment of momentum in the recirculation bubble. 
N. A. Siddiqui and M. A. chaab / JAFM, Vol. 14, No. 1, pp. 147-164, 2021.

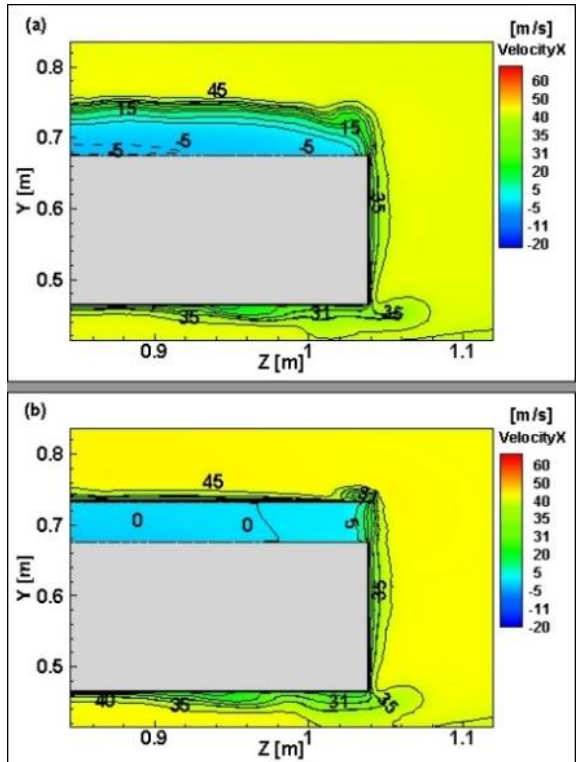

Fig. 16. Velocity $X$ contours at the $X=0.67$ (a) Base model (b) $\alpha=10^{\circ}$ (Dashed lines represent the negative velocities).

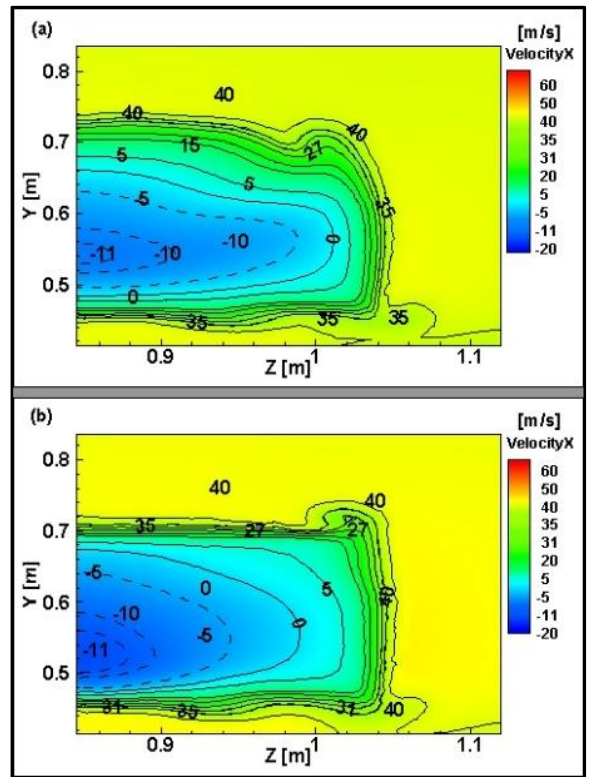

Fig. 17. Velocity $X$ contours at the $X=0.87 \mathrm{~m}$ (a) Base model (b) $\alpha=10^{\circ}$ (Dashed lines represent the negative velocities).

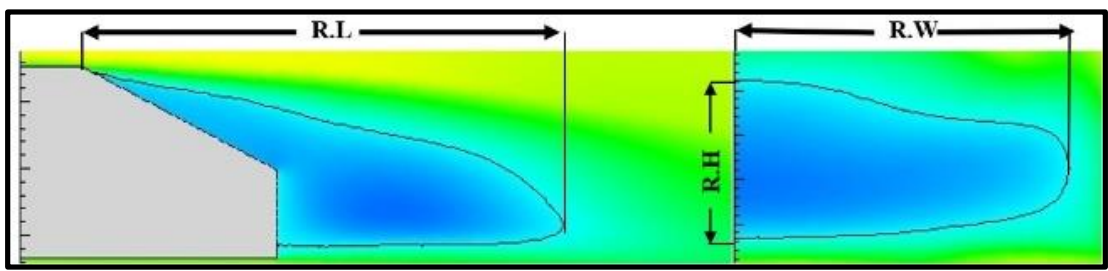

Fig. 18. Nomenclature of the recirculation region.
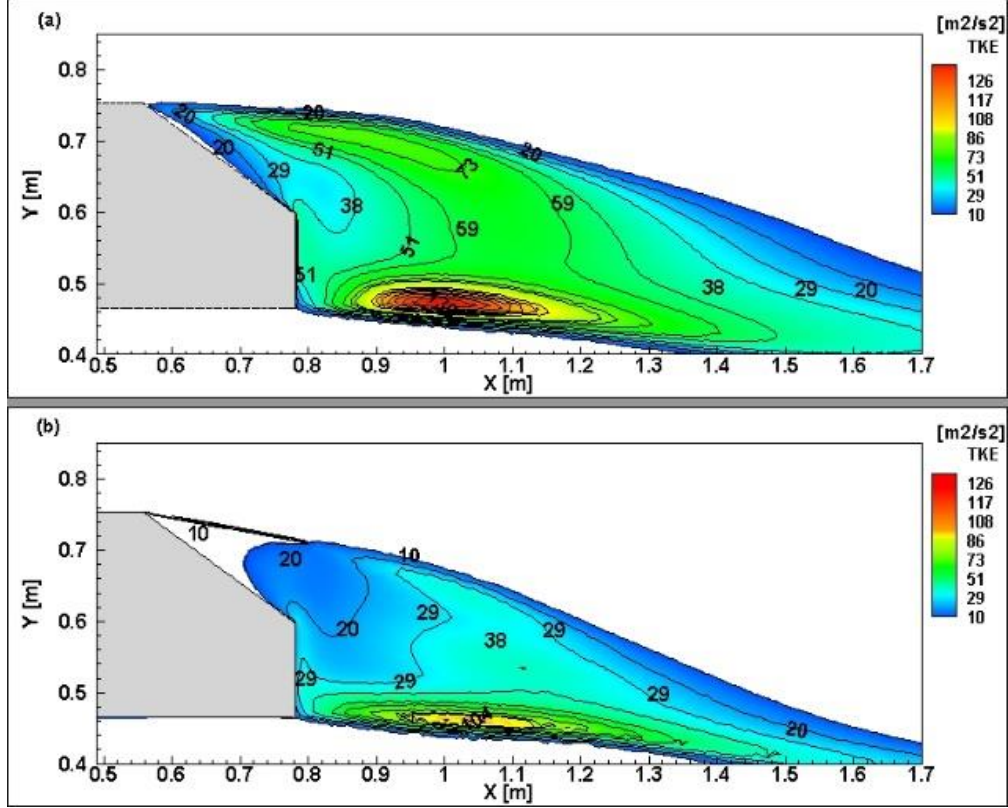

Fig. 19. Turbulent Kinetic Energy at the $Z=0.8438 m$ (a) Base model (b) $\alpha=10^{\circ}$.

In the current investigation, Figs. 14-17 show the recirculation length, width and velocity field at the recirculation region. They are shown in Fig. 18 and documented in Table 2 below. The velocity field captures a reduced velocity in a $10^{\circ}$ flap case, which is almost zero inside the slant volume and reduced in 

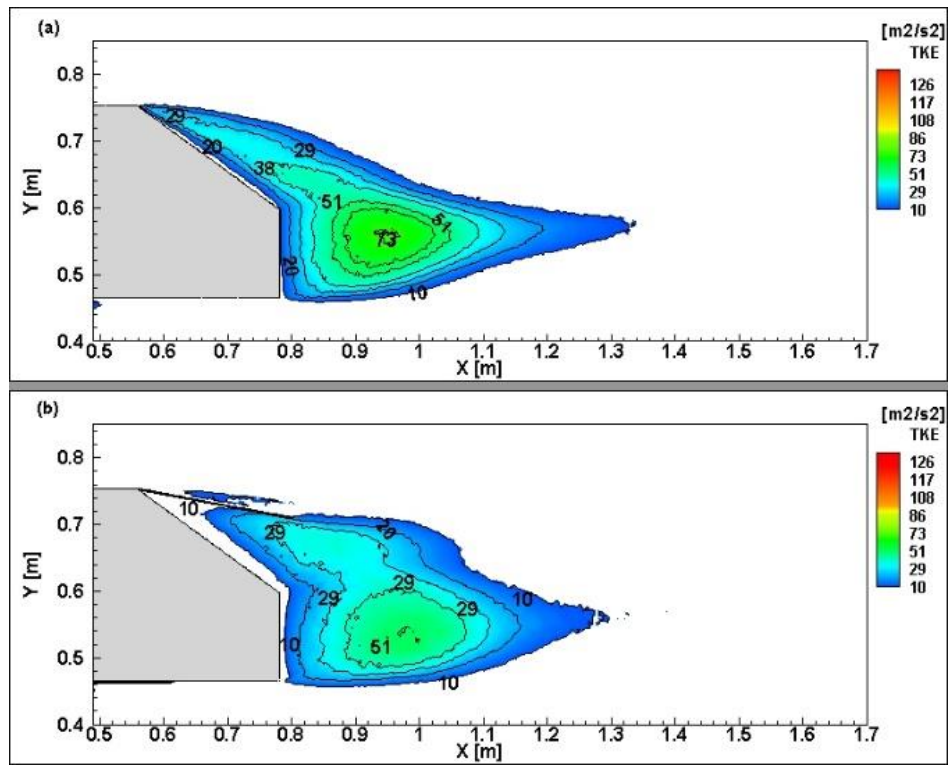

Fig. 20. Turbulent Kinetic Energy at the $Z=1.03$ (a) Base model (b) $\alpha=10^{\circ}$.

the wake. This reduced fluctuation of the velocity is related to the mean drag value highlighted by (Mariotti and Buresti, 2013). Hence the results of the current study support the view described by (Mariotti et al. 2015) and previous related work (Mariotti and Buresti 2013) that reduced velocity fluctuations leading to base drag reduction by extending the recirculation region.

Moreover, Figs. 19 and 20 show the features of turbulent kinetic energy (TKE). It reports a reduced TKE for the $10^{\circ}$ case inside the slant volume and within the recirculation bubbles. Therefore, the present study is supported by the proposal of (Barros et al. 2016) that the reduction in TKE, lessens the wake entrainment and thus increases the recirculation region and reduces the wake width as well.

The effect of modifying the spanwise wake or the overall aspect ratio of the wake is also confirmed by the (Mariotti et al. 2019). They argued that the grooves provided a higher resistance to flow separation and delayed it. This led to a narrower wake in the lateral direction. This view resonates with the finding of the current investigation. The recirculation region has been quantified in Table 2 (and Fig. 18) that shows an $18.1 \%$ increment in the recirculation length and a $40 \%$ reduction in the wake width. The pressure analysis in Figs. 9-10 revealed that the decrease in drag is due to the flap, which delayed the separation. This delay in flow separation reduces the size of the separation bubbles, as discussed with respect to Figs. 14-17. Although the method of separation delay is different compared to the grooves used by (Mariotti et al. 2019), the effect is the same - separation delay leading to a decrease in velocity fluctuations and turbulent kinetic energy. This influences the wake aspect ratio, as highlighted by Barros et al. 2016. Therefore, the conclusion in the present study is consistent with that of Mariotti et al. (2019).
Overall, the transfer of the separation bubbles outside the slant volume due to the passive flap modifies the backflow region. The mechanism of the collapse of the backflow before the slant surface allows a pressure recovery that is not available in the base case. The modification corroborates this pressure recovery in the aspect ratio of the wake. Therefore, it is evident that the mechanism of pressure recovery is the direct exposure of the flow around the base where the backflow breaks down.

Table 2 Specification of the recirculation region

\begin{tabular}{|c|c|c|c|}
\hline Specification & Base Model & $10^{\circ}$ flap & \% Change \\
\hline R.L $(\mathrm{m})$ & 0.55 & 0.65 & $18.1 \%$ \\
\hline$R . W(\mathrm{~m})$ & 0.175 & 0.105 & $40 \%$ \\
\hline$R . H(m)$ & 0.19 & 0.19 & 0 \\
\hline
\end{tabular}

\subsection{Turbulent Kinetic Energy}

The drag reduction mechanism due to suppressed bubbles at the slanted surface also changes the turbulence parameters, as discussed above. The

turbulent kinetic energy is one of the parameters that is considered to connect the above drag reduction mechanisms.

Turbulence kinetic energy (TKE) manifests the mean kinetic energy available per unit mass of the turbulent eddies(Hinze 1975). Figures 19 and 20 captures the variation in the TKE for the base and $10^{\circ}$ case. For the base case, the first maxima of the TKE begin at the flow separation point as the ejected shear layer is highly unstable and creates energetic eddies (Habchi et al. 2015). This turbulent strength of 20$30 \mathrm{~m}^{2} / \mathrm{s}^{2}$ dominates the slant surface. However, in the $10^{\circ}$ case, the TKE inside the slant volume is reduced to $10 \mathrm{~m}^{2} / \mathrm{s}^{2}$. It also shows that unlike the base model, 


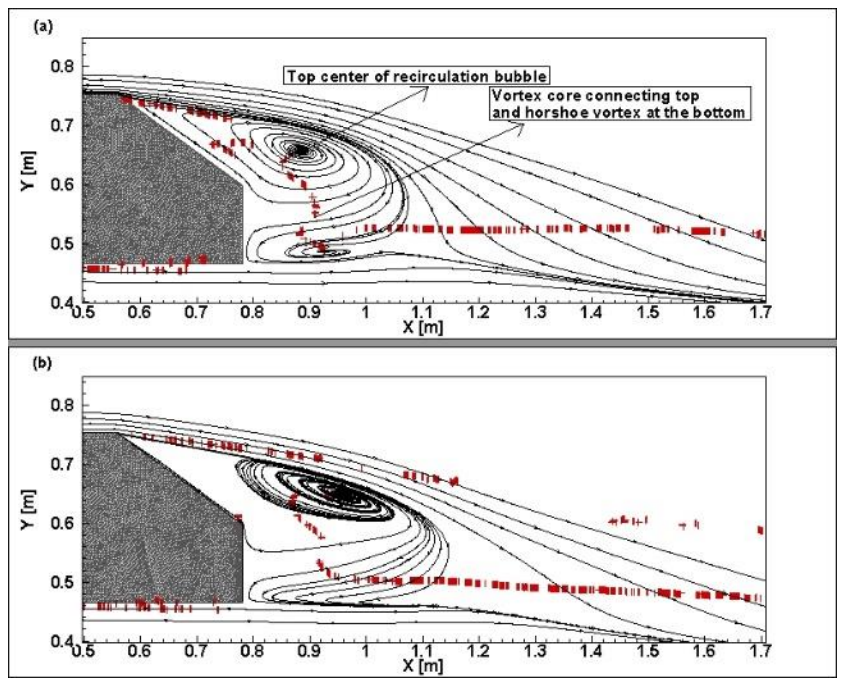

Fig. 21. Structure of the vortex core with the velocity streamlines at Symmetry plane.

the entire slant volume has the same TKE of $10 \mathrm{~m}^{2} / \mathrm{s}^{2}$. Additionally, the vertical base also captures a reduction in the TKE. It shows an increase at the ground side due to the interaction of eddies in the downstream locations. The contribution made by the longitudinal vortices is depicted at $\mathrm{Z}=1.03 \mathrm{~m}$ plane Fig. 19. It shows the TKE of $10 \mathrm{~m}^{2} / \mathrm{s}^{2}$ inside the slant volume, which increases to $20 \mathrm{~m}^{2} / \mathrm{s}^{2}$ at the end of slant volume. Nonetheless, these values are comparatively less then what is observed in the base case. Moreover, the highest amount of TKE is concentrated around $\mathrm{X}=0.95 \mathrm{~m}$ in both the case. For the $10^{\circ}$ angle, at this location the TKE is reduced which ultimately reduced the surrounding TKE effect.

The strength of the turbulent flow is reduced due to the flap because it shifts the major separation bubbles behind the vertical base. From the Kolmogorov energy cascade, eddy size differentiates the energy storage, and therefore, large eddies have superior energy. It is since the length scale of the eddies is proportional to the turbulent kinetic energy, which is called the integral scale of turbulence (Tennekes and Lumley 1972). Therefore, it suggests that at the flap angle $10^{\circ}$, the energy available to eddies is less compared to the base case and hence the size of eddies too. Consequently, the $10^{\circ}$ case provides a less turbulent flow at the slant volume, and that is consistent at the downstream locations as well. It suggests that the addition of a flap makes a flow less turbulent and prior disbursement compared to the base model (Barros et al. 2016) states this reduction in TKE lessens the wake entrainment which led to the enlarged recirculation region. Therefore, the addition of a flap reduced the base drag is a function of the recirculation region aspect ratio is re-stressed by the current investigation. This relation is already highlighted in section 4.4.

\section{VORTEX CORE AND DYNAMICS OF THE WAKE}

The idea behind the vortex formation and its relation to the base drag due to the separation, is supported by the development of the vortex core location. The extraction of vortex core rests on identifying the centre of swirling flow in a three-dimensional discretized vector field. It is based on the critical point theory developed by (Sujudi and Haimes 1995). The vortex core captures the centreline around which a vortex is formed.

The structure of the vortex core is shown along with the velocity streamline in Figs. 21 and 22 . The vortex core shows a connection between the upper separation bubble and horseshoe vortex at the vertical base for the base case model Figs. 21 (a) and 21(b). It travels from the top bubble to the bottom and then extends longitudinally. However, the addition of a flap first shifts the location of the top separated bubble and reduced the length of the bubble as well. The pressure recovery emphasizes this physical modification of the separation bubbles discussed in Fig. 9. The cross-section of the vortex core (Fig. 22) captures the differences in the wake width, which is reduced in the case of a $10^{\circ}$ case. Also shown in Figs. 16-18 in the section on velocity analysis. Moreover, Fig. 22 (b) shows that the formation of the spanwise wake is not uniform for a $10^{\circ}$ flap; instead, it breaks down in the middle of the vertical base at the bottom side, unlike the base model. It suggests that the formation of horseshoe vortices influences wake width reduction. The existence of modified horseshoe vortex is consequently correlated to the modified spanwise wake formation. This correlation between horseshoe vortex and the wake width needs to be further studied. Nonetheless, the figures also depict the formation of C-vortex which is further identified with the vortex identification method in the next section.

\section{VORTEX IDENTIFICATION}

The wake behind the models is dominated by the vorticity-carrying free-shear layer and the associated vortical structures. These are developed due to 
inviscid instability mechanisms (Caylan 2019; McAuliffe and Yaras 2008). Vortex identification is a method to visualize these vortical structures behind the model. There are several methods to capture vortices (V Holmén, 2012). One of the essential and critical means of vortex identification is the Qcriterion proposed by (Hunt et al. 1988). It is based on the tensor of the velocity gradient $\nabla u$ which is segregated into one symmetric strain tensor $S_{i j}$ and one anti-symmetric rotational part $\Omega_{i j}$. This is described as per Eq. 4 (Gohlke et al. 2008)

$\nabla_{u}=u_{i j}=S_{i j}+\Omega_{i j}$

$\mathrm{S}_{\mathrm{ij}}=\frac{1}{2\left(\mathrm{u}_{\mathrm{i}, \mathrm{j}}+\mathrm{u}_{\mathrm{i}, \mathrm{i}}\right)}$

$\Omega_{i j}=\frac{1}{2\left(u_{i, j}-u_{j, i}\right)}$

Equation (4) is satisfied by the eigenvalues of $\nabla u$ is given by:

$$
\begin{gathered}
\sigma^{3}-P \sigma^{2}+Q \sigma-R=0 \\
P=u_{i, j}=0, \quad Q=\frac{1}{2\left(u_{i, i}^{2}-u_{i, j} u_{i, j}\right)}, \\
R=\operatorname{det}\left(u_{i, j}\right)
\end{gathered}
$$
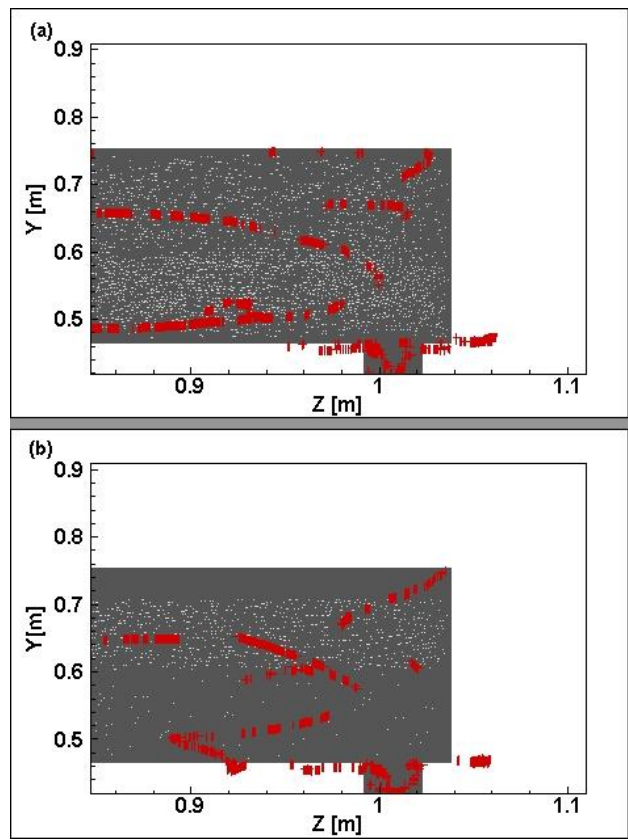

Fig. 22. Cross-section view of the vortex core.

The Q-criterion is based on the second invariant of the velocity gradient denoted as Q. It also includes the condition that ambient pressure should be higher than the pressure of vortex to separate it. The full dynamics is presented in Eq. (8):

$Q=\frac{1}{2}\left(u^{2}{ }_{i, i}-u_{i, j} u_{i, j}\right)=-\frac{1}{2} u_{i, j} u_{i, j}=\frac{1}{2}\left(\left\|\Omega^{2}\right\|-\right.$ $\left.\left\|\mathrm{S}^{2}\right\|\right)$

Q is a local term for balance vorticity magnitude $\Omega$ and strain rate $\mathrm{S}$. By putting $\mathrm{Q}>0$, it is possible to disintegrate the regions where the strength of vorticity surpasses the strain rate. Since within a lowpressure tube with small cross-section engulfed by isobaric layers of pressure, Laplacian will be positive if the flow has a uniform density. This variable is related to the Q, the second invariant, and Q must be positive (Dubief and Delcayre 2000; Hunt et al. 1988; Jeong and Hussain 1995). The Q-criterion for the base and $10^{\circ}$ case is shown in Fig. 23.
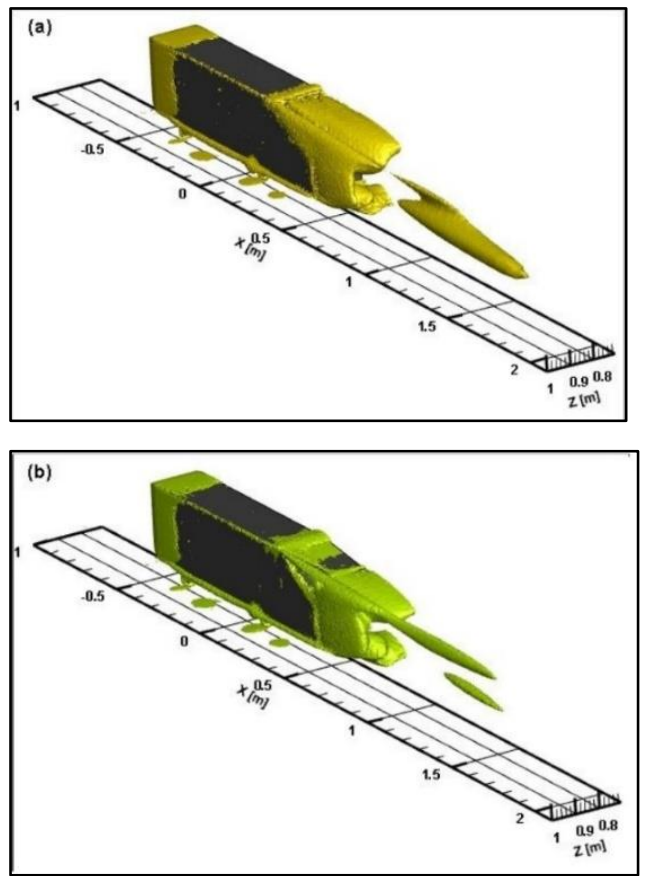

Fig. 23. $Q$ criterion of velocity invariant with $\mathrm{Q} / \mathrm{Uref}=150$ (a) Base model (b) $\alpha=10^{\circ}$.

The large scale vortical structures enclosed the entire slant surface in the base case, whereas the intensity of vortex formation is less in the $10^{\circ}$ case. The base case (Fig. 21a) shows a large vortex at the vertical base and around the recirculation region. Nonetheless, these large eddies broke down around $\mathrm{X}=0.7 \mathrm{~m}$ and formed a longitudinal vortex that goes beyond the length $X=1.5 \mathrm{~m}$.

In the $10^{\circ}$ case, the vortex development at the slanted surface is less compared to the base case, which supports a lower fluctuation of the turbulent flow. It also shows a developed longitudinal vortex that maintains a reduced vortical structure but lost its intensity before the $X=1.5 \mathrm{~m}$. It means that the kinetic energy available to the eddies is reduced earlier. Similarly, the vortex radius is also lower in the $10^{\circ}$ case, which shows the dispersion of the vortex, unlike in the base model. Its physics is already discussed in the drag mechanism section

The kinetic energy of these vortex structures are compared in Figs. 24. At $X=0.8 \mathrm{~m}$, the kinetic energy is the same for both cases, along with the height of the vertical base. However, at the slanted surface, the kinetic energy begins to diverge and is higher in the base case than the $10^{\circ}$ case. The reason behind this sudden divergence is the effect of delay in the separation that shifts the recirculation bubbles. Therefore at $\mathrm{X}=0.9 \mathrm{~m}$, this divergence between the base and $10^{\circ}$ case becomes more evident. That shows 


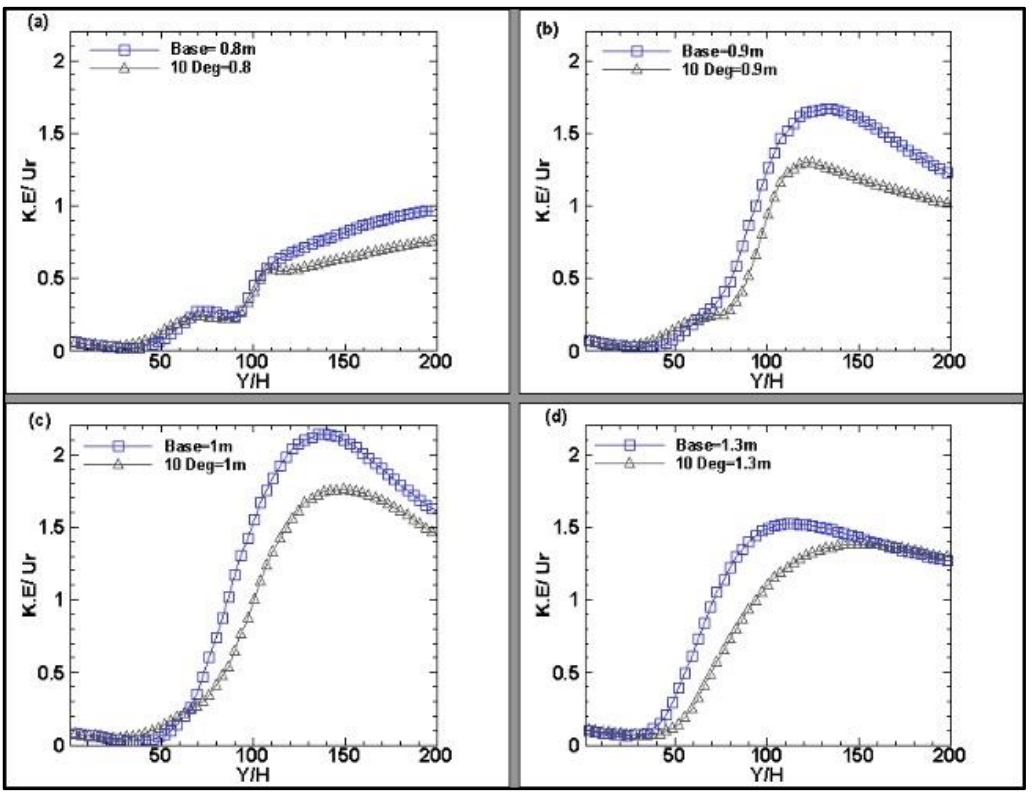

Fig. 24. Comparison of the Kinetic energy (K.E/Uref) for the base and $\alpha=10^{\circ}$ case where (a) data at $X$ $=0.8 \mathrm{~m}$ (b) data $X=0.9 \mathrm{~m}$ (c) data at $X=1.0 \mathrm{~m}$ and $(\mathrm{d})$ data $\mathrm{Xt}=1.3 \mathrm{~m}$. The data is extracted through a vertical line shown in Fig. 9 at the symmetry plane.

the energy associated with the $10^{\circ}$ case tends to decay even from the base position at $X=0.8 \mathrm{~m}, 0.9 \mathrm{~m}$, and $1.0 \mathrm{~m}$. At these locations, the kinetic energy is the same for the base and $10^{\circ}$ case near the ground, but however, it differs at the upper side in Fig. 21a,b,c. At $X=1.3 \mathrm{~m}$, where the large vortical structures are visible in both the cases (Fig. 23), the kinetic energy differs between the base and $10^{\circ}$ case. The kinetic energy available to the $10^{\circ}$ case is lower than the base case, as shown in Fig. 24d. Therefore the vortex structure of the $10^{\circ}$ case disburses before the base case model. It validates the results of the turbulent kinetic energy as well that provided a less turbulent flow with a $10^{\circ}$ case suggesting earlier disbursement. The fluctuations in the kinetic energy confirm the idea of reduced velocity fluctuations leading to reduced wake entrainment (Barros et al. 2016). More precisely, the location of horseshoe vortices at $\mathrm{X}=0.9$, the divergence between the base and $10^{\circ}$ begins just above the ground where its existence was found in Figs. 21 and 22 . The $10^{\circ}$ case has lower kinetic energy at that region and this difference enlarges as it moves towards the upper separation bubble. As reported and discussed regarding Figs. 14-17, the recirculation region for the $10^{\circ}$ case extend up to $X=1.2 \mathrm{~m}$ and the profiles of kinetic energy show that throughout the recirculation region the velocity fluctuations and hence the energy available to the eddies is less for the $10^{\circ}$ case which contributes to the pressure recovery and drag reduction. Therefore, the current investigation supports the investigation of (Barros et al. 2016; Mariotti et al. 2015, 2019) and others on the same relation between base drag and recirculation region.

\section{CONCLUSIONS}

A numerical simulation is carried out on a proposed rectangular flap as a simple passive device installed at the slant surface of a $35^{\circ}$ Ahmed model. By changing the flap angle, the slant volume is controlled to investigate the effect on the aerodynamic drag. It is found that a rectangular flap provides some drag reduction when the flap angle is varied from $0^{\circ}$ to $25^{\circ}$ at $5^{\circ}$ intervals. The flap angle of $10^{\circ}$ was found to provide the highest drag reduction of approximately $14 \%$. To confirm that the $10^{\circ}$ drag reduction is indeed the highest, additional angles of $7^{\circ}$ and $13^{\circ}$ angles were also analyzed and verified as such. The reasons and mechanism of drag reduction were investigated further. Analysis of flow features, along with vortex identification, revealed that the achieved drag reduction is due to the suppression of the bubbles generated inside the slant volume and vertical base. The flap was also proved to delay flow separation and displaced the separation bubbles behind the vertical base, which has been attributed to the drag reduction through the change in the wake aspect ratio. The flap shortens the backflow region, namely, recirculation length and width, that contributes to drag reduction. However, no reduction in the vertical height of the wake is found. The study of turbulent kinetic energy reveals that the $10^{\circ}$ case makes the flow less chaotic, which facilitates the reduction in wake entrainment leading to extended recirculation region. Also, the kinetic energy fluctuations re-stressed the relation between drag reduction and extension of the recirculation region. The current results is in agreement with the existing investigations as well but with a very simple passive device of flap. Moreover, the research suggests that this passive device does not influence much the induced drag due to the side edges. Therefore, it does not contribute to the pressure recovery at the slant volume. Further investigations by focusing on the slant volume with new active or passive devices will reveal more insights. 


\section{REFERENCES}

Ahmed, S. R., G. Ramm and G. Faltin (1984). Some Salient Features of The Time-Averaged Ground Vehicle Wake. SAE Transactions 93(2), 473-503.

Altaf, A., Omar, A. A. and W. Asrar (2014a). Passive drag reduction of square back road vehicles. Jnl. of Wind Engineering and Industrial Aerodynamics 134, 30-43.

Altaf, A., Omar, A. A. and W. Asrar(2014b). Review of passive drag reduction techniques for bluff road vehicles. IIUM Engineering Journal 15(1), 61-69.

Ashton, N., A. West, S. Lardeau and A. Revell (2016). Assessment of RANS and DES methods for realistic automotive models. Computers and Fluids 128, 1-15.

Barros, D., J. Borée, B. R. Noack, A. Spohn and T. Ruiz (2016). Bluff body drag manipulation using pulsed jets and Coanda effect. Journal of Fluid Mechanics 805, 422-459.

Beaudoin, J. F. and J. L. Aider (2008). Drag and lift reduction of a 3D bluff body using flaps. Experiments in Fluids 44(4), 491-501.

Capone, A. and G. P. Romano (2019). Investigation on the effect of horizontal and vertical deflectors on the near-wake of a square-back car model. Journal of Wind Engineering and Industrial Aerodynamics 185, 57-64.

Caylan, U. (2019). Computations and Measurements of the Effects of Trailing-Edge Geometry on the Wake of Bluff Bodies.

Choi, H., J. Lee and H. Park (2014). Aerodynamics of Heavy Vehicles. Annual Review of Fluid Mechanics 46(1), 441-468.

Choi, H., W.-P. Jeon and J. Kim (2008). Control of Flow Over a Bluff Body. Annual Review of Fluid Mechanics 40(1), 113-139.

Dubief, Y. and F. Delcayre (2000). On coherentvortex identification in turbulence. Journal of Turbulence 1, N11.

Emery, C., Z. Liu, A. G. Russell, M. T. Odman, G. Yarwood and N. Kumar (2017). Recommendations on statistics and benchmarks to assess photochemical model performance. Journal of the Air \& Waste Management Association 67(5), 582-598.

Fernholz, H. H. and F. Urzynicok (2006). Control of weak and strong reverse-flow regions in incompressible turbulent boundary layers. Lecture Notes in Control and Information Sciences 330, 1-44.

Fourrié, G., L. Keirsbulck, L. Labraga and P. Gilliéron (2011). Bluff-body drag reduction using a deflector. Experiments in Fluids 50(2), 385-395

Gerrard, J. H. (1966). The mechanics of the formation region of vortices behind bluff bodies. Journal of Fluid Mechanics 25(2), 401413.

Gohlke, M., J. F. Beaudoin, M. Amielh, and F. Anselmet (2008). Thorough analysis of vortical structures in the flow around a yawed bluff body. Journal of Turbulence 9 , N15.

Grandemange, M., M. Gohlke and O. Cadot (2013). Turbulent wake past a three-dimensional blunt body. Part 1. Global modes and bi-stability. Journal of Fluid Mechanics 722, 51-84.

Griffin, O. M. and M. S. Hall (1991). Review-vortex shedding lock-on and flow control in bluff body wakes. In Journal of Fluids Engineering, Transactions of the ASME 113(4), 526-537).

Guilmineau, E. (2008). Computational study of flow around a simplified car body. Journal of Wind Engineering and Industrial Aerodynamics 96(6-7), 1207-1217.

Guilmineau, E., Deng, G. and J. Wackers (2011). Numerical simulation with a DES approach for automotive flows. Journal of Fluids and Structures 27(5-6), 807-816.

Guilmineau, E., G. B. Deng, A. Leroyer, P. Queutey, M. Visonneau and J. Wackers (2016). Assessment of RANS and DES methods for the Ahmed body. ECCOMAS Congress 2016 Proceedings of the 7th European Congress on Computational Methods in Applied Sciences and Engineering 1(June), 912-921.

Ha, J., S. Jeong and S. Obayashi (2011). Drag reduction of a pickup truck by a rear downward flap. International Journal of Automotive Technology 12(3), 369-374.

Habchi, C., T. Lemenand, D. Della Valle, A. Al Shaer and H. Peerhossaini (2015). Experimental study of the turbulent field behind a perforated vortex generator. Journal of Applied Mechanics and Technical Physics 56(4), 569-579.

Han, T. (1989). Computational analysis of threedimensional turbulent flow around a bluff body in ground proximity. AIAA Journal 27(9), 1213-1219.

Hinterberger, C., M. García-Villalba and W. Rodi (2004). Large eddy simulation of flow around the Ahmed body (pp. 77-87). Springer, Berlin, Heidelberg.

Hinze, J. O. (1975). Turbulence. (2nd Revise). McGraw-Hill College.

Holmén, V. (2012). Methods for vortex identification. In Master's Theses in Mathematical Sciences. http://lup.lub.lu.se/ student-papers/record/3241710

Hunt, J. C. R., A. A. Wray and P. Moin (1988). Eddies, streams, and convergence zones in turbulent flows. https://www. semanticscholar.org/paper/Eddies\%2Cstreams $\% 2 \mathrm{C}$-and-convergence-zones-in-turbulentHunt 
N. A. Siddiqui and M. A. chaab / JAFM, Vol. 14, No. 1, pp. 147-164, 2021.

Wray/4e1d2fdcd44cc416e44b6186c1f7c23cc1 $200 \mathrm{~b} 7 \mathrm{c}$.

Hsu, F. H., \& Davis, R. L. (2010). Drag reduction of Tractor-trailers using optimized add-on devices. Journal of Fluids Engineering, Transactions of the ASME, 132(8). https://doi.org/10.1115/1.4001587.

Hucho, W., \& Sovran, G. (1993). Aerodynamics of Road Vehicles. Annual Review of Fluid Mechanics, 25(1), 485-537.

Igali, D., O. Mukhmetov, Y. Zhao, S. C. Fok and S. L. The (2019). Comparative Analysis of Turbulence Models for Automotive Aerodynamic Simulation and Design. International Journal of Automotive Technology 20(6), 1145-1152.

Jeong, J. and F. Hussain (1995). On the identification of a vortex. Journal of Fluid Mechanics 285(1), 69.

Kim, D., H. Lee, W. Yi and H. Choi (2016). A bioinspired device for drag reduction on a threedimensional model vehicle. Bioinspiration \& Biomimetics 11(2), 026004.

Lanfrit, M. (2005). Best practice guidelines for handling Automotive External Aerodynamics with FLUENT. In Fluent Deutschland GmbH (Vol. 2).

Lienhart, H., C. Stoots and S. Becker (2002). Flow and Turbulence Structures in the Wake of a Simplified Car Model (Ahmed Modell). In New Results in Numerical and Experimental Fluid Mechanics III (pp. 323-330). Springer Berlin Heidelberg.

Makowski, F. T. and S. E. Kim (2000, March 6). Advances in External-Aero Simulation of Ground Vehicles Using the Steady RANS Equations, SAE Technical Paper 2000-010484.

Mariotti, A. (2018). Axisymmetric bodies with fixed and free separation: Base-pressure and nearwake fluctuations. Journal of Wind Engineering and Industrial Aerodynamics 176, 21-31

Mariotti, A., G. Buresti and M. V. Salvetti (2015). Connection between base drag, separating boundary layer characteristics and wake mean recirculation length of an axisymmetric bluntbased body. Journal of Fluids and Structures 55, 191-203.

Mariotti, A., G. Buresti and M. V. Salvetti (2019). Separation delay through contoured transverse grooves on a 2D boat-tailed bluff body: Effects on drag reduction and wake flow features. European Journal of Mechanics, B/Fluids 74 , 351-362.

Mariotti, A., G. Buresti, G. Gaggini and M. V. Salvetti (2017). Separation control and drag reduction for boat-tailed axisymmetric bodies through contoured transverse grooves. Journal of Fluid Mechanics 832, 514-549.

Mariotti, Alessandro and G. Buresti (2013). Experimental investigation on the influence of boundary layer thickness on the base pressure and near-wake flow features of an axisymmetric blunt-based body. Experiments in Fluids 54(11), 1-16.

McAuliffe, B. R. and M. I. Yaras (2008). Numerical study of instability mechanisms leading to transition in separation bubbles. Journal of Turbomachinery 130(2).

Menter, F. R. (1994). Two-equation eddy-viscosity turbulence models for engineering applications. AIAA Journal 32(8), 1598-1605

Mukut, A. N. M. M. I. and M. Z. Abedin (2019). Review on Aerodynamic Drag Reduction of Vehicles. International Journal of Engineering Materials and Manufacture 4(1), 1-14.

Pavia, G., M. Passmore and M. Varney(2019). Lowfrequency wake dynamics for a square-back vehicle with side trailing edge tapers. Journal of Wind Engineering and Industrial Aerodynamics 184, 417-435.

Pujals, G., S. Depardon and C. Cossu (2010). Drag reduction of a 3D bluff body using coherent streamwise streaks. Experiments in Fluids 49(5), 1085-1094.

Roshko, A. (1955). On the Wake and Drag of Bluff Bodies. Journal of the Aeronautical Sciences 22(2), 124-132.

Sujudi, D. and R. Haimes (1995). Identification Of Swirling Flow In 3-D Vector Fields. American Institute of Aeronautics and Astronautics, 1-8.

Tennekes, H. and J. L. Lumley (1972). A first course in turbulence. The MIT Press.

Tian, J., Y. Zhang, H. Zhu and H. Xiao (2017) Aerodynamic drag reduction and flow control of Ahmed body with flaps. Advances in Mechanical Engineering 9(7), 1-17.

Zhang, C., C. P. Bounds, L. Foster and M. Uddin (2019). Turbulence Modeling Effects on the CFD Predictions of Flow over a Detailed FullScale Sedan Vehicle. Fluids 4(3), 148. 